\title{
Floristic Diversity in the Lake Cluster of Pokhara Valley, Central Nepal
}

Hom Nath Pathak ${ }^{1}$ 凶, Bharat Babu Shrestha ${ }^{2}$, Dinesh Raj Bhuju ${ }^{3}$ and Prabin Bhandari ${ }^{4}$

${ }^{1}$ Central Department of Botany, Tribhuvan University, Kathmandu, Nepal ${ }^{2}$ Prithvi Narayan Multiple Campus, Tribhuvan University, Pokhara, Nepal

${ }^{3}$ Resources Himalaya Foundation, Kathmandu, Nepal

${ }^{4}$ State Key Laboratory of Systematic and Evolutionary Botany, Institute of Botany,

Chinese Academy of Sciences, Beijing, 10009 China

${ }^{5}$ University of Chinese Academy of Sciences, Beijing, 100049 China

homnpathak@gmail.com

\begin{abstract}
Wetlands support exceptionally high biodiversity and provide valuable ecosystem services, yet they are among the most threatened habitats due to anthropogenic activities. Conservation and management planning of wetlands requires, among others, a comprehensive floristic account. In this study, we prepared a checklist of the flowering plants found in the wetlands of the Lake Cluster of Pokhara Valley (LCPV), a Ramsar site of Nepal, located in a rapidly urbanizing capital city of Gandaki Province in Central Nepal. Voucher specimens were collected from the study sites through multiple visits during the monsoon (June-August) and autumn (September-November) seasons. Species were categorized based on their life forms (Raunkiaer's classification) and native distribution range (native, naturalized, invasive). Ethno-botanical uses of the plant species were compiled from the published literature. We identified 230 plant species belonging to 70 families and 177 genera. Asteraceae ( 25 species), Poaceae (22 species), Fabaceae (18 species), Cyperaceae (16 species), and Lamiaceae (11 species) were species-rich families. Therophytes (30\%) were the dominant life form followed by Hemicryptophytes (27\%). Among 230 species, 183 species were native and 47 species naturalized; among the naturalized species, 21 species were invasive. Most of the plant species (61\%) have medicinal values while others have food (24\%) and fodder values (13\%).
\end{abstract}

Keywords: invasive alien species, Ramsar site, Raunkiaer's life form, wetland flora 


\section{INTRODUCTION}

Wetlands are areas of marsh, fen, peatland, or sea, whether natural or artificial, permanent or temporary, with fresh, brackish, or saltwater that is stagnant or flowing, including areas of marine water not exceeding six meters in depth at low tide (Ramsar Convention Secretariat, 2013). Wetlands are also called nature's supermarkets as they provide diverse goods and services to mankind and kidney of nature as they purify and filter water (Mandal \& Mukherjee, 2012). For example, 21 ethnic communities out of 101 in Nepal depend on wetland resources for their subsistence (Lamsal et al., 2014). Wetlands are among the most productive life support systems in the world which are also immensely important for mankind as they provide humanity the freshwater supply, food and construction materials, and biodiversity, flood control, groundwater recharge, and climate change mitigation(Halls, 1997, https://www.ramsar.org). Wetlands that are the habitat of threatened species and provide valuable ecosystem services are designated as a Ramsar site under the Ramsar Convention on Wetlands (https:/www.ramsar.org/). There are over 2400 Ramsar sites in the world including 10 sites in Nepal. The total area covered by all Ramsar sites in the world exceeds 2.5 million square kilometers (https://www.ramsar.org/about/wetlands-of-international-importance-ramsar-sites). Ramsar sites in Nepal occupy 605.61 square kilometers (MoFE, 2018a).

Wetlands support exceptionally high biodiversity and provide habitats to both water and land organisms (Denny, 1994). Floral diversity constitute a major resource in wetlands. A prerequisite to understanding the ecosystem type and biodiversity pattern of the region is a knowledge of the floral diversity of any region (Singh et al., 2017). Such floristic data will be useful for tracking changes in the pattern of vegetation in the future. In Nepal, the analysis of wetland flora is limited. A specific gap exists for the floristic studies in LCPV. Ethno-botanical studies of plants and plant products are essential for proper management of plant resources, in addition to the flora research (Cunningham, 2001).

Lake Cluster of Pokhara Valley (LCPV) is the most recently declared Ramsar site of Nepal (MoFE, 2018b). The LCPV includes nine lakes located in Pokhara Metropolitan, a rapidly urbanizing city of touristic attraction in Central Nepal. This study was carried out to prepare a checklist of the flowering plants found in the LCPV and analyzes their 
taxonomic diversity, life forms, biogeographic status, and ethnobotanical uses. The results of this study are useful for the management and conservation planning of the LCPV.

\section{Study area}

The study was carried out in the Lake Cluster of Pokhara Valley (LCPV) which includes nine lakes located within Pokhara Metropolitan City, central Nepal (fig. 1). The Valley is located between $27^{\circ} 55^{\prime}-28^{\circ} 23^{\prime} \mathrm{N}$ latitude and $83^{\circ} 48^{\prime}-84^{\circ} 11^{\prime} \mathrm{E}$ longitude, with an area of 133.41 sq. km in Pokhara Metropolitan City (Shrestha \& Kshetri, 2008). Pokhara is one of the most popular tourist destinations in Nepal, and a provincial capital of Gandaki Province. Of the nine lakes (table. 1), Phewa, Begnas, Rupa, and Deepang are visited by tourists frequently for natural beauty, fishing, and boating. The lakes and other water bodies are important sources of drinking water, fishery, irrigation, and hydropower (MoFE, 2018b). Besides they provide recreational, religious, spiritual, and inspirational values to local people and tourists.

TABLE 1. General information on the Lake Cluster of Pokhara Valley (MoFE, 2018b).

\begin{tabular}{|c|c|c|c|c|c|c|c|c|c|c|}
\hline SN & Attributes & $\frac{\pi}{2}$ & 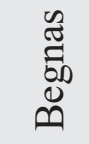 & $\underset{\overparen{Z}}{\stackrel{\pi}{二}}$ & 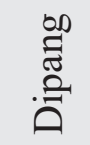 & $\frac{\bar{\pi}}{\bar{\pi}}$ & 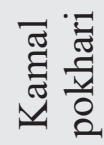 & 节 & $\begin{array}{l}\bar{\Xi} \\
\overline{0} \\
\overline{0}\end{array}$ & 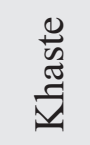 \\
\hline 1 & $\begin{array}{c}\text { Ward } \\
\text { number }\end{array}$ & 6 & 31 & 33 & 27 & 28 & 13 & 26 & 26 & 26 \\
\hline 2 & $\begin{array}{c}\text { Area } \\
\text { occupied by } \\
\text { water }\left(\mathrm{Km}^{2}\right)\end{array}$ & 4.33 & 3.13 & 1.11 & 0.14 & 0.007 & 0.013 & 0.08 & 0.027 & 0.13 \\
\hline 3 & $\begin{array}{l}\text { Catchment } \\
\text { area }\left(\mathrm{Km}^{2}\right)\end{array}$ & 119.39 & 18.6 & 26.02 & 2.39 & 1.6 & 1.35 & 0.61 & 0.18 & 2.69 \\
\hline 4 & $\begin{array}{l}\text { Lowest } \\
\text { elevation } \\
\text { (m asl) }\end{array}$ & 763 & 647 & 580 & 687 & 672 & 822 & 741 & 742 & 739 \\
\hline
\end{tabular}




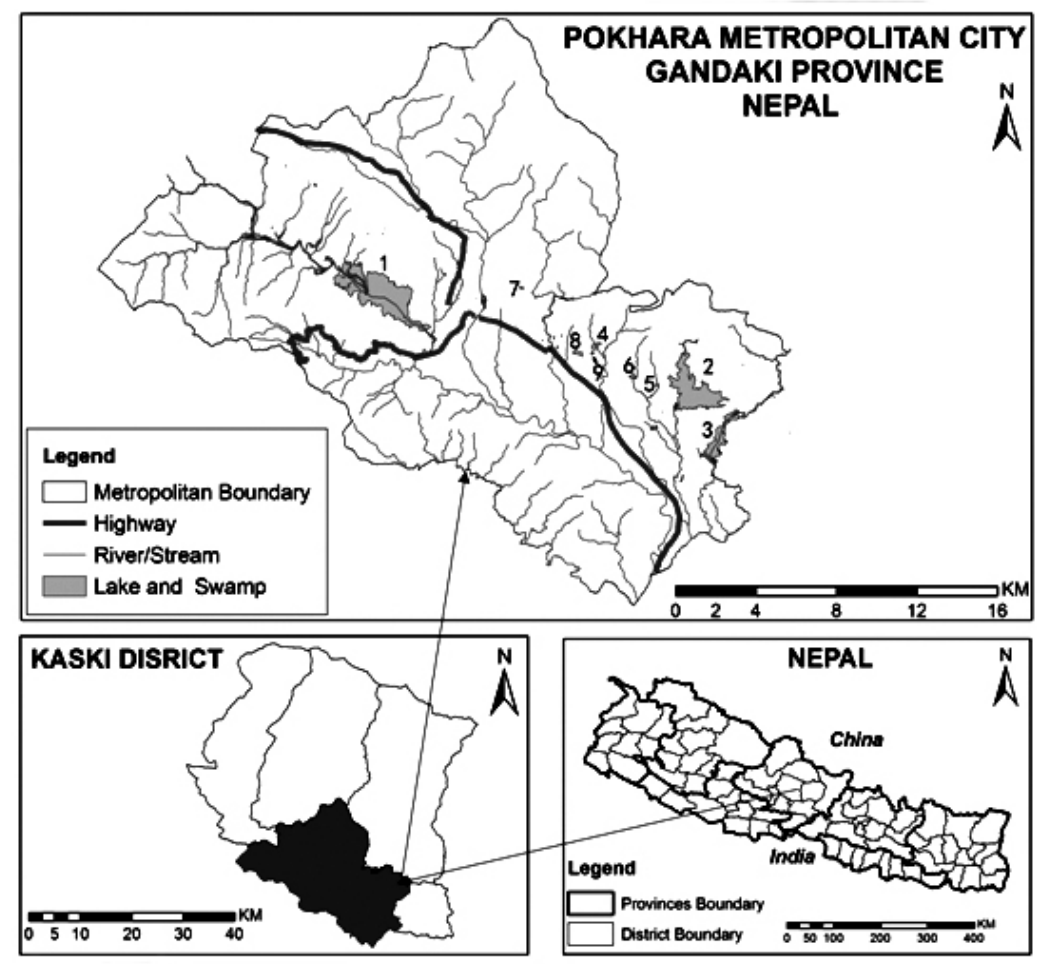

Fig.1. Lake Cluster of Pokhara Valley, Kaski District, Gandaki Province, Nepal. (Numerical values in Pokhara Metropolitan City map represent different lakes: 1. Phewa, 2. Begnas, 3. Rupa, 4. Khaste, 5. Maidi, 6. Deepang, 7. Kamalpokhari, 8. Gunde, 9. Niureni).
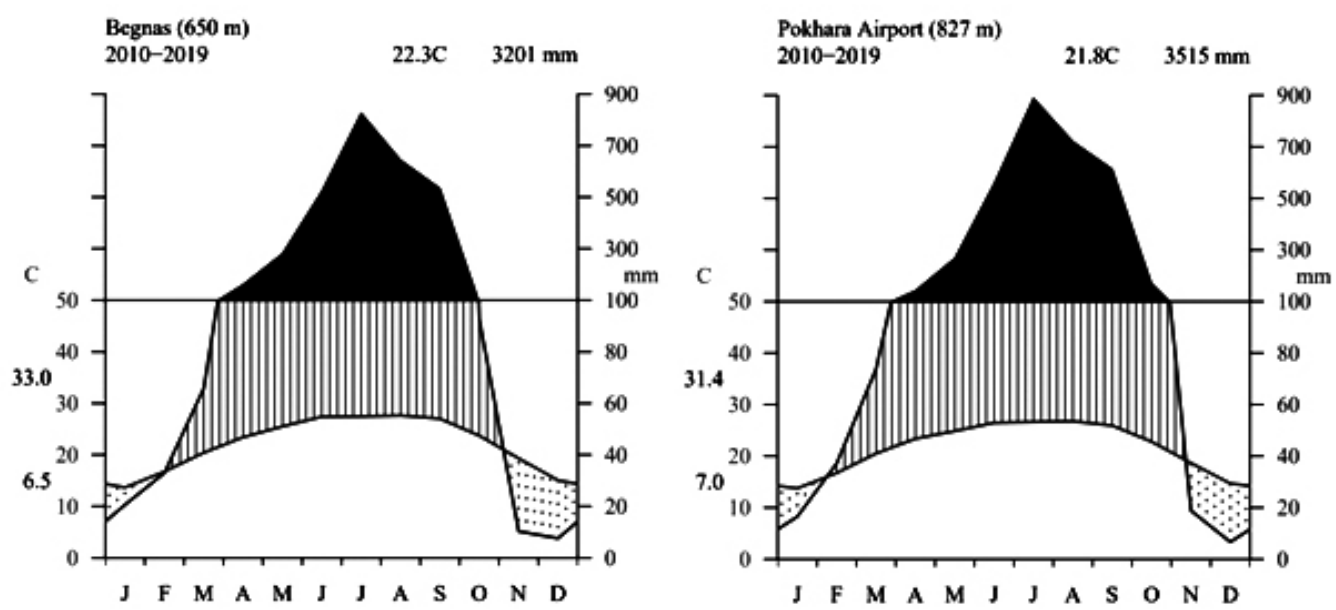

Fig.2. Ombrothermic diagram of the climatic data between2010 to 2019 at Begnas and Pokhara airport. 
Pokhara Valley lies in a subtropical region dominated by Schima-Castanopsis vegetation, while small patches of the riverine forest dominated by Acacia catechu, Alnus nepalensis, Pandanus furcatus are also found in the gorges of Seti and other associated rivers. The minimum temperature was recorded at $6.5^{\circ} \mathrm{C}$ at the Begnas weather station and $7^{\circ} \mathrm{C}$ at the Pokhara airport. Similarly, the maximum temperature recorded at Begnas was $33^{\circ} \mathrm{C}$ and $31.4^{\circ} \mathrm{C}$ at the airport. The mean annual temperatures were $22.3^{\circ} \mathrm{C}$ and $21.8^{\circ} \mathrm{C}$, respectively at Begnas andthe airport. Similarly, average annual precipitation was $3201 \mathrm{~mm}$ and $3515 \mathrm{~mm}$ at Begnas and Pokhara airport respectively (fig. 2). Dry periods are from midNovember to mid-February and the wet periods from mid-February to mid-November. The extreme wet periods extend between March to October and peak in June/July.

\section{METERIALS AND METHODS}

\section{Specimen collection and identification}

Lakes of LCPV were visited for six times from June 2018 to December 2020, representing two different seasons: monsoon (June-August) and autumn (September-November) because they are the peak flowering seasons of the wetland flora. Voucher specimens of flowering plant species were collected along the shore, inside the lakes, and around the lakes. The free-floating and submerged species growing within the territory of the lakeshore were collected following Haynes (1974). The collected specimens were pressed and dried using newspaper and herbarium pressure. The prepared specimens were identified consulting regional and national floras (Grierson \& Long, 19832001; Wu et al., 1994-2008; Watson et al., 2011). During the identification process, the collected samples were also cross-checked with the specimens available at the National Herbarium and Plant Laboratories (KATH) and Tribhuvan University Central Herbarium (TUCH). For some doubtful species, expert views were also considered. The nomenclature of the families followed APG-IV (Angiosperm Phylogeny Group, version IV) (Chase et al., 2016), while the nomenclature of the genera and species followed Roskov et al. (2020). Identified specimens were deposited in TUCH and KATH.

\section{Species categorization and ethnobotanical use}

Habits of the species (herb, shrub or tree;annual or perennial) were determined based on field observations of the flora (Grierson \& Long, 1983-2001, Wu et al., 1994-2008, Watson et al., 2011). The life forms of the identified species were classified following 
Raunkiaer's classification as Phanerophytes (Perennating buds from aerial parts more than $2 \mathrm{~m}$ from the soil surface), Chamaephytes (Perennating buds on aerial parts less than $2 \mathrm{~m}$ from the soil surface), Hemicryptophytes (Buds at ground level), Cryptophytes (Buds below ground or water), and Therophytes (Plants that survive unfavourable conditions as seeds) (Kent, 2012). The native ranges were extracted from Wu et al. (1994-2008) and Plants of the World Online (2020) (http://www.plantsoftheworldonline.org/); for some species with uncertain native range, experts' advice was also solicited. Continents (e.g. Europe, Americas) of the native distribution range of the naturalized alien species were identified. Some of the naturalized alien species were categorized as 'invasive' following the list of invasive alien plants of Nepal by Shrestha (2019). International Union for Conservation of Nature (IUCN) threatened categories were identified for each species (www.iucnredlist.org). The collected plant species were compared with the list of species reported by the Ministry of Forests and environment (MoFE, 2018b) from the LCPV. Ethnobotanical uses of the collected plant species in Nepal were obtained from the following references: Manandhar (2002), Sah et al .(2002), Bishokarma et al. (2005), Baral \& Kurmi (2006), Acharya (2009), Joshi \& Joshi (2009), Kunwar et al. (2010), Niroula \& Singh (2011), Lamsal et al. (2014), Kunwar et al. (2015), Adhikari et al. (2019), Budha-Magar et al. (2020), Bhatt \& Kunwar, (2020), and Sharma et al. (2020). Uses of the plant species were grouped into the following categories: medicines, food, forage, fodder, fiber yielders, rituals plants, fish poisons, timber yielders, fuel-woods, ornamentals, dye yielders, construction materials, green manures, hedge plants, tannin yielders, soaping agents and fermenting agents. Use percent was calculated by dividing the number of times the plant used (e.g. as medicine) by total plant species enumerated (e.g. 216) multiplied by hundred. Due to multiple uses of single plant species, a sum of the percentage values of the different use categories was more than $100 \%$.

\section{RESULTS AND DISCUSSION}

Altogether 230 flowering plant species were collected belonging to 177 genera and 70 families from the LCPV (Appendix I). Among them, 168 species were dicots and 62 species monocots. Out of 177 genera, 34 genera had two or more than two species while the rest of others was represented by a single species. Asteraceae ( 25 species), Poaceae (22 species), Fabaceae (18 species), Cyperaceae (16 species) and Lamiaceae (11 species) were the species-rich families (table 2). Analysis of the floristic composition 
of the LCPV revealed Asteraceae and Poaceaeae as the dominant families, which agree with the general floral composition of Nepal (Press et al., 2000). Poaceae was also reported as a dominant family in the wetlands of other parts of Nepal (e.g. Chitwan by Dangol et al., 2014). In Nepal, the analysis of wetland flora is limited. Sah et al. (2002) reported altogether 401 plant species belonging to 264 genera under 84 families from the Ghodaghodi lake area. Altogether 115 species belonging to 45 families were recorded from the wetland flora of Rupandehi district (Sharma et al., 2019). A total of 108 plant species were recorded from the wetland and periphery of Raja-Rani Tal, Morang (Sharma et al., 2020). Dangol et al. (2014) documented 117 plant species belonging to 39 families and 92 generain the area of Rampurghol, Chitwan. Compared to the list of 436 plant species reported in the management plan of the LCPV (MoFE, 2018b), this research revealed the presence of 155 additional plant species suggesting that previous floristic studies of the LCPV are far from complete.Regarding habits, 167 species were herbs, 38 shrubs and 25 trees. Out of the total, 76 were annuals and 154 perennials.

Table 2. Number of species recorded from the Lake Cluster of Pokhara Valley that belong to different families.

\begin{tabular}{|c|l|c|}
\hline S.N. & \multicolumn{1}{|c|}{ Family } & Number of species \\
\hline 1. & Asteraceae & 25 \\
2. & Poaceae & 22 \\
3. & Fabaceae & 18 \\
4. & Cyperaceae & 16 \\
5. & Lamiaceae & 11 \\
6. & Polygonaceae, Rubiaceae & 8 \\
7. & Malvaceae & 6 \\
8. & Acanthaceae, Euphorbiaceae, Moraceae, Orchidaceae & 5 \\
9. & Amaranthaceae, Commelinaceae, Rosaceae, Solanaceae, & 4 \\
10. & Urticaceae & Hypericaceae, Phyllanthaceae, \\
11. & Apocynaceae, Araceae, Boraginaceae, Caryophyllaceae, & 3 \\
& Convolvulaceae, Dioscoreaceae, Fagaceae, Lauraceae, & \\
& Linderniaceae, Lythraceae, Melastomataceae, Myrtaceae, & 2 \\
& Oleaceae, Onagraceae, Oxalidaceae, Plantaginaceae, & \\
12 & Pontederiaceae, Primulaceae, Vitaceae & 1 \\
\hline
\end{tabular}


The IUCN red list category of the plant species were attempted to find. Out of the 230 species, 67 plant species were under the Least Concern category and the rest of the others(163 species) were not assessed to any threat category. None of the plants were government protected. However, the ecosystems maintained by these plant species are the habitat of several threatened animal species (MoFE, 2018b).

The majority of the species were native ( 183 species) while 47 species were naturalized alien species. Out of them, 21 naturalized species were invasive (table 3). Though the flora of LCPV was dominated by the native species, the presence of naturalized species contributing to one-fifth of the total flora recorded during the present study could not be underestimated. Furthermore, globally worst invasive species such as Eicchornia crassipes, Chromolaena odorata, Lantana camara and Mikania micrantha (Lowe et al., 2000) had already invaded LCPV. The majority of the naturalized species (81\%) and all of the invasive alien species were native of the Americas (fig. 3, table 3). The dominance of the American native plant species in the naturalized flora has been also reported at the national level (Bhattarai et al., 2012).

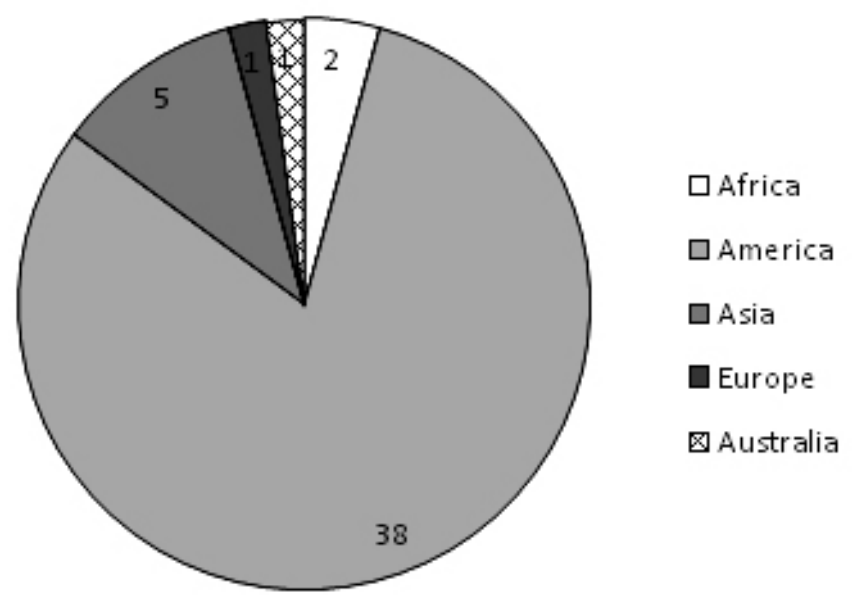

Fig. 3. Number of naturalized species with their place of origin. 
Journal of Natural History Museum Volume 31, 2019-20 9

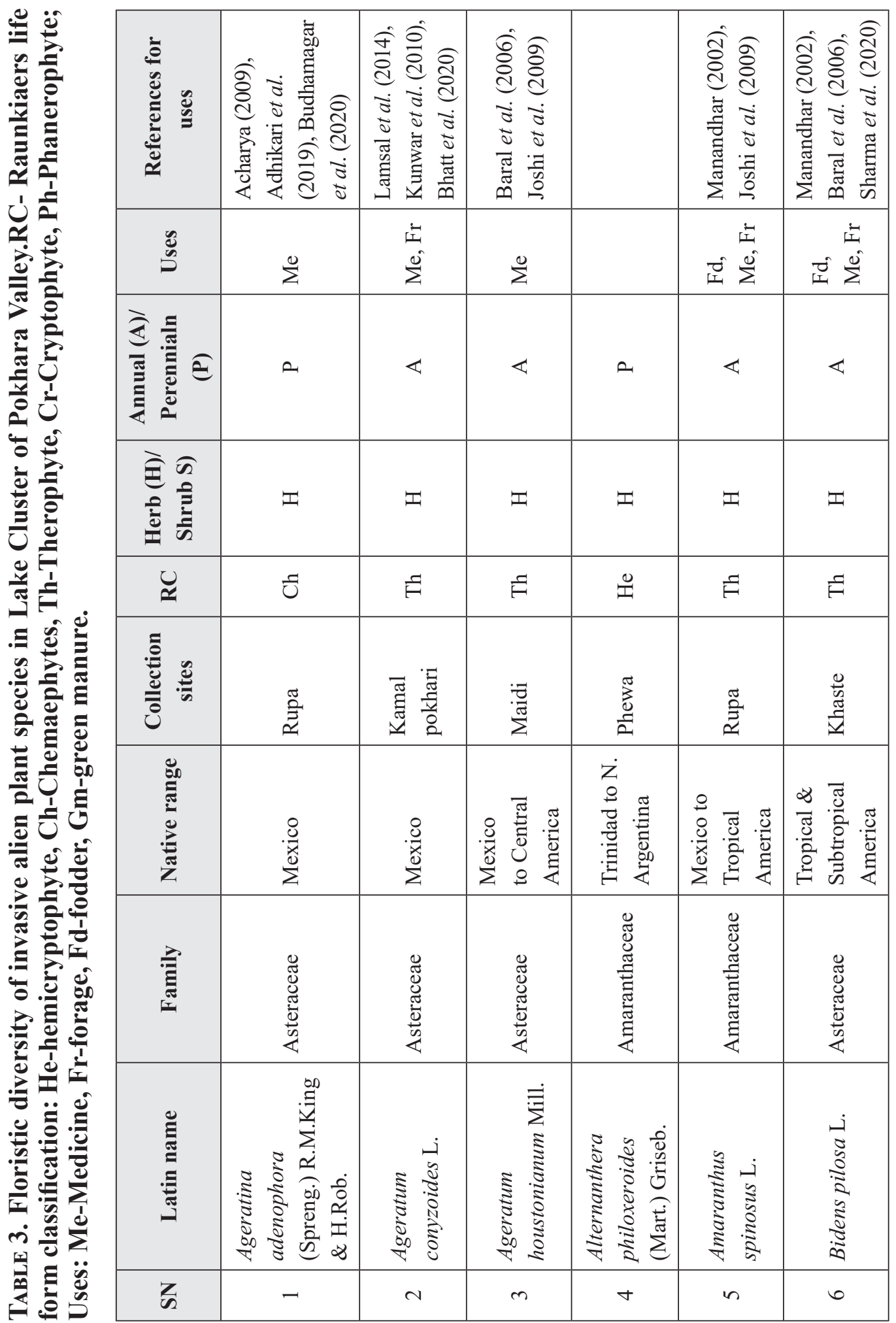




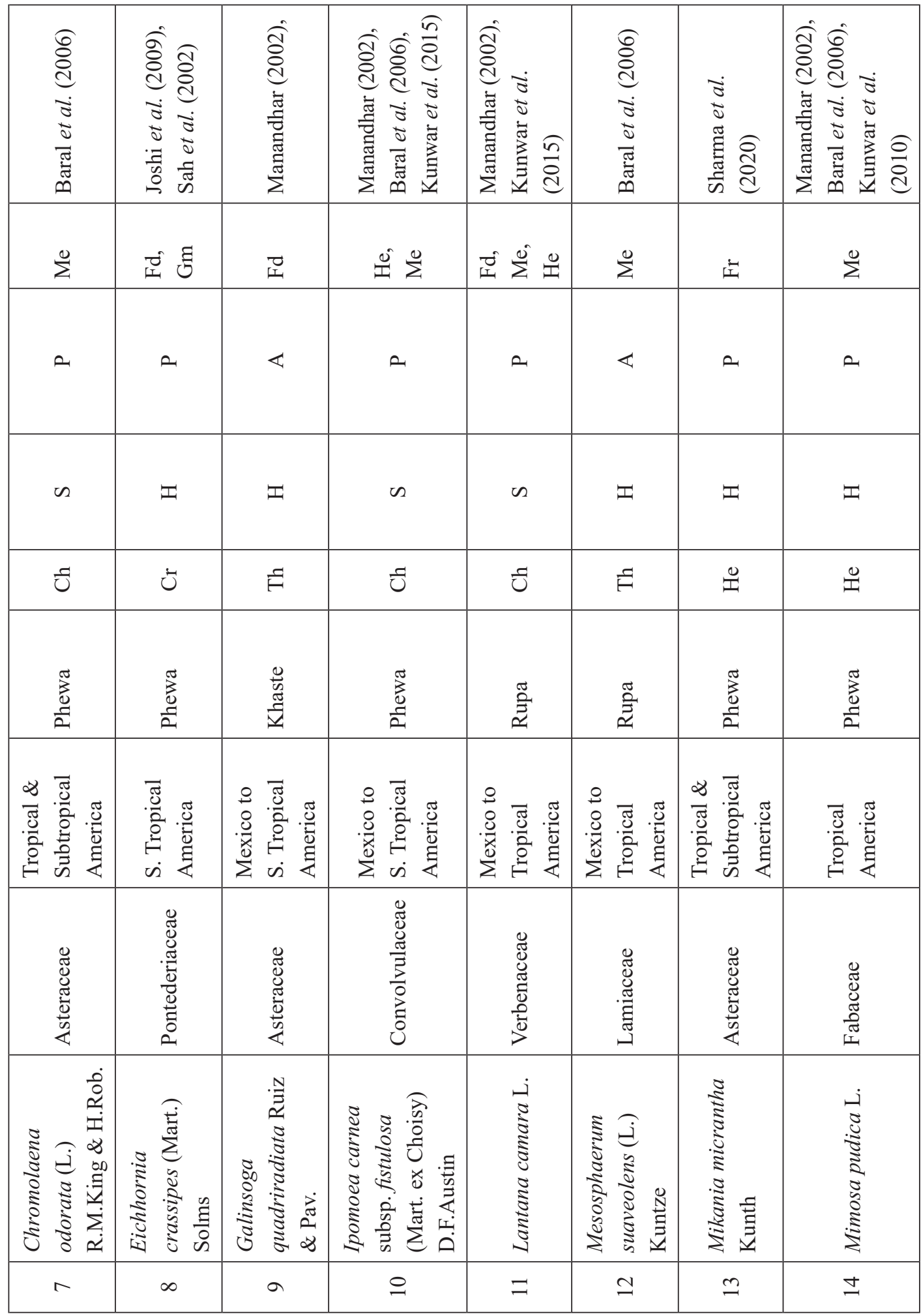




\begin{tabular}{|c|c|c|c|c|c|c|}
\hline & & & 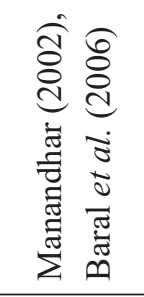 & 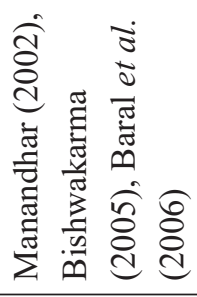 & & 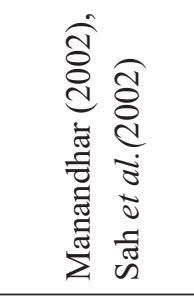 \\
\hline & & & 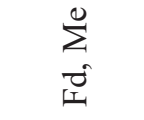 & $\sum_{i}^{0}$ & $\sum^{0}$ & $\sum_{\Sigma}^{0}$ \\
\hline$a$ & $\ll$ & $a$ & $\varangle$ & $\ll$ & $\rho_{1}$ & $\varangle$ \\
\hline$I$ & $I$ & $I$ & $I$ & $I$ & $I$ & $I$ \\
\hline$\dot{U}$ & 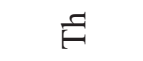 & $\dot{U}$ & 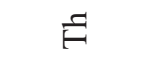 & $\tilde{F}$ & $\stackrel{\mathscr{I}}{ \pm}$ & $\tilde{F}$ \\
\hline 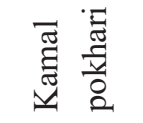 & $\frac{\pi}{2}$ & $\frac{\pi}{0}$ & $\frac{\pi}{2}$ & $\frac{\pi}{0}$ & $\frac{\pi}{2}$ & $\underset{\widetilde{2}}{\stackrel{\Xi}{二}}$ \\
\hline 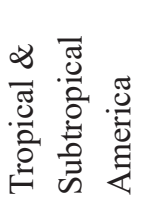 & 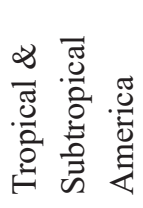 & 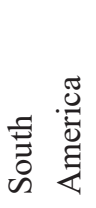 & 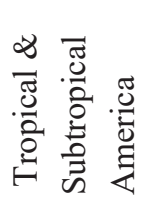 & 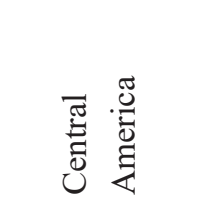 & 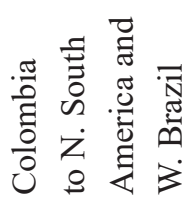 & 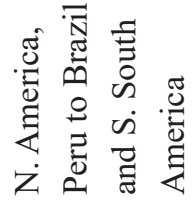 \\
\hline 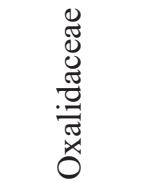 & 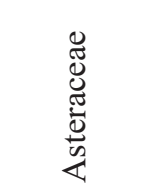 & 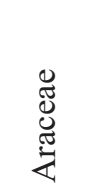 & 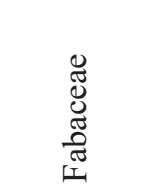 & 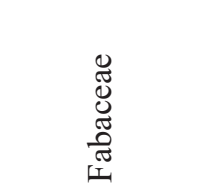 & 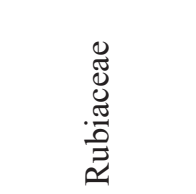 & 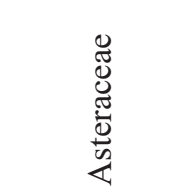 \\
\hline 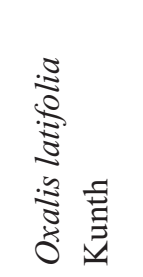 & 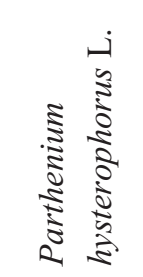 & 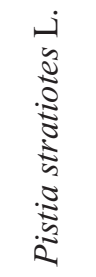 & 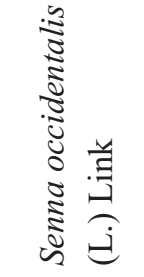 & 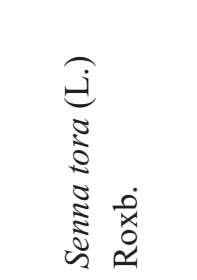 & 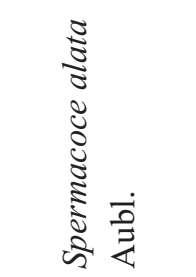 & 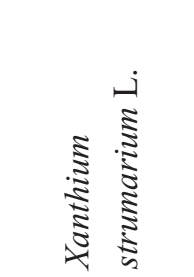 \\
\hline$\stackrel{n}{=}$ & $\underline{\sigma}$ & I & $\infty$ & 2 & $\stackrel{\sim}{ }$ & $\vec{\sim}$ \\
\hline
\end{tabular}


The life form of dominant plant species is one of the basic physiognomic attributes (Beard, 1978), which show the plant-environment interaction, and it helps to understand the micro and macroclimate under which plant species flourish (Khan et al., 2018). The vegetation in our study area ranged from phanerophytes to therophytes with the dominance of therophytes and hemicryptophytes (fig. 4). The hemicryptophytes and cryptophytes constituted the species of the herb. The chamaephytes observed were mainly woody shrub species while the phanerophytes constituted the trees and the associated epiphytes and climbers, growing in the adjoining areas of wetlands. Hydrophytes (a component of Cryptophytes) constituted 10 species; common among them were Trapa natans, Eichhormia crassispes, Pistia stratiotes, Hydrilla verticillata, Leersia hexandra and Ludwigia adscendens. Such dominancy of hydrophytes was also reported from a study in wetlands of Central Nepal (Burlakoti \& Karmacharya, 2004).

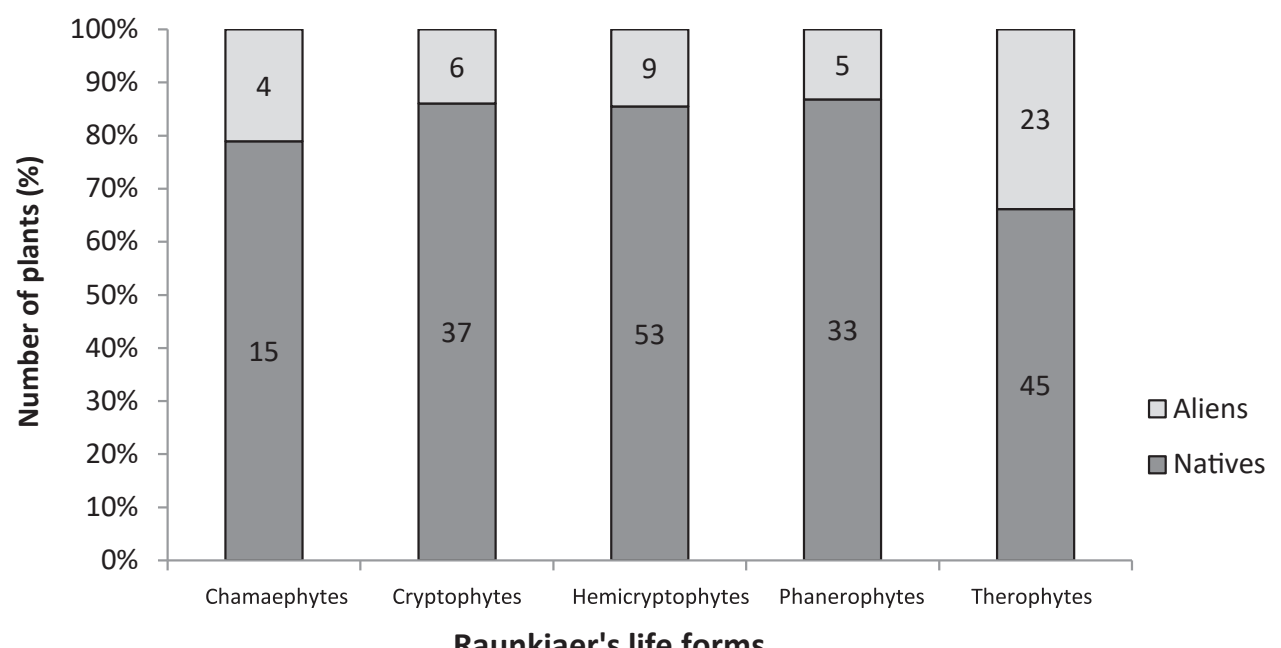

Raunkiaer's life forms

Fig. 4. Percentage of native and naturalized aline plant species in each life form. Values inside the bars represents the number of species.

A review of the previous studies revealed that about $71 \%$ (166 species) of the flowering plants recorded in the LCPV have one or moreuse values (table 4). Most of them (59\%) were used as medicines, followed by food (25\%) and forage and fodder (13\%). This suggests that the LCPV provides important provisioning services to the people living in the landscape surrounding the LCPV. A similar type of research was reported by Sah et al. (2002) in the Ghodaghodi lake area and Dangol (2014) in Rampur Ghol, Chitwan. 
Table 4. Uses of the plant species reported from the LCPV. Uses of the species have been compiled from the previous studies (See Appendix I and II for details).

\begin{tabular}{|c|l|c|c|}
\hline S.N. & \multicolumn{1}{|c|}{ Use category } & Number of species & Percentage of species \\
\hline 1. & Medicinal uses & 141 & 61.30 \\
\hline 2. & Food value & 57 & 24.78 \\
\hline 3. & Fodder and forage & 31 & 13.47 \\
\hline 5. & Fiber yielders & 8 & 3.47 \\
\hline 6. & Rituals plants & 4 & 1.73 \\
\hline 7. & Fish poisons & 4 & 1.73 \\
\hline 8. & Timber yielders & 3 & 1.30 \\
\hline 9. & Fuelwood & 2 & 0.86 \\
\hline 10. & Ornamental plants & 2 & 0.86 \\
\hline 11. & Dye yielders & 2 & 0.86 \\
\hline 12. & Use in construction & 2 & 0.86 \\
\hline 13. & Green manure & 1 & 0.43 \\
\hline 14. & Hedge plant & 1 & 0.43 \\
\hline 15. & Tannin yielders & 1 & 0.43 \\
\hline 16. & Soaping agent & & 0.43 \\
\hline 17. & Fermenting agent & 1 & 0.43 \\
\hline & & 1 & \\
\hline
\end{tabular}

This study gives an insight into floral diversityin the LCPV, their biogeography, as well as their uses. Most of the species were native, perennial, and Therophytes. The LCPV provides habitat for several plant species with medicinal values. The presence of several invasive alien plant species, some of them being globally worst, suggests that the wetland habitats of LCPV have been degrading. Management responses to plant invasions, together with other drives of degradation, will help to restore the habitats and ensure the continuous supply of ecosystem services. This will provide both economic as well as conservation benefits. 


\section{ACKNOWLEDGEMENTS}

We are thankful to Keshab Raj Rajbhandari (National Herbarium and Laboratories, KATH), Sangeeta Rajbhandary (Central Department of Botany, Tribhuvan University, CDB TU), Suresh Kumar Ghimire (CDB, TU), Deepak Pant (CDB, TU), Yadu Paudel, Hem Raj Paudel (National Herbarium and Plant Laboratories, Godawari, KATH), Ganga Datt Bhatt (KATH), and Reeta Chhetri (KATH) for their help in plant identification. We are also thankful to Subhash Khatri (KATH) for providing facilities at KATH to compare the herbarium specimens. This research was made possible from the fellowship awarded to the first author (HNP) from the Rector's office, TribhuvanUniversity, Nepal.

\section{REFERENCES}

ACHARYA, K P (2009) Utilization and status of plant resources in northern Part of Pokhara Valley, Central Nepal. Ethnobotanical leaflets 13:116-122.

ADHIKARI, M; THAPA, R; KUNWAR, R M; DEVKOTA, H P; POUDEL, P (2019) Ethnomedicinal Uses of Plant Resources in the Machhapuchchhre Rural Municipality of Kaski District, Nepal. Medicines 6(2), 69. DOI: 10.3390/ medicines6020069

BARAL, S R; KURMI, P P (2006) A compendium of medicinal plants in Nepal. Rachana Sharma, Kathmandu, Nepal.

BHATT, M D; KUNWAR, R M (2020) Distribution pattern and ethnomedicinal uses of plants in Kanchanpur district, Far-Western Nepal. Ethnobotany Research and Applications 20: 1-21.

BHATTARAI, K R, MÅREN, I E, SUBEDI, S C (2014) Biodiversity and invasibility: Distribution patterns of invasive plant species in the Himalayas, Nepal. Journal of Mountain Science 11(3): 688-696. DOI: 10.1007/s11629-013-2821-3.

BISHOKARMA, B K; KINSEY, C K; DANGOL, D. R; CHAUDHARY, P (2005) Folk use of plant resource at Madi valley of Chitwan District, Nepal. Banko Janakari 15(2): 28-33.

BUDHA-MAGAR, S; BHANDARI, P; GHIMIRE, S K (2020) Ethno-medicinal survey of plants used by Magar (Kham) community, Rolpa district, Western Nepal. Ethnobotany Research and Applications 19: 1-29. 
CHASE, M W; CHRISTENHUSZ, M J M; FAY, M F; BYNG, J W; JUDD, W S; SOLTIS, D E, ... STEVENS, P F (2016) An update of the Angiosperm Phylogeny Group classification for the orders and families of flowering plants: APG IV. Botanical Journal of the Linnean Society 181(1): 1-20.

CUNNINGHAM, A B (2001) Applied ethnobotany: people, wild plant use and conservation. Earthscan, London, United Kingdom.

DANGOL, D R; GAUTAM, B; OLI, B B (2014) Wetland plants and their local uses: Observations from Rampur Ghol, Chitwan, Nepal. Journal of Natural History Museum 28:142-159.

DENNY, P (1994) Biodiversity and wetlands. Wetlands Ecology and Management, 3(1): 55-611.

GRIERSON, AJC ; LONG,DG (1983-2001)Flora of Bhutan. Royal Botanic Garden, Edinburgh, United Kingdom.

HALLS, A J E (1997) Wetlands, biodiversity and the Ramsar convention: the role of the convention on wetlands in the conservation and wise use of biodiversity. In Ramsar Convention Bureau, Gland, Switzerland (Vol. 13).

HAYNES, R R (1984) Techniques for collecting aquatic and marsh plants. Annals of the Missouri Botanical Garden 71(1): 229-231.

JOSHI, A R; JOSHI, K (2009) Indigenous Uses of Wetland Plant Diversity of two Valleys (Kathmandu and Pokhara) in Nepal. Ethnobotany 21: 11-17

KENT, M (2012) Vegetation description and data analysis A practical approach. Wiley Blackwell, A John Wiley \& Sons, Ltd, Publication, Oxford, UK

KHAN, W; KHAN, S M; AHMAD, H; ALQARAWI, A A; SHAH, G M; HUSSAIN, M; ABD-ALLAH, E (2018) Life forms, leaf size spectra, regeneration capacity and diversity of plant species grown in the Thandiani forests, district Abbottabad, Khyber Pakhtunkhwa, Pakistan. Saudi Journal of Biological Sciences 25(1): 94-100.

KUNWAR, R M; ACHARYA, R P; CHOWDHARY, C L; BUSSMANN, R W (2015) Medicinal plant dynamics in indigenous medicines in far-west Nepal. Journal of Ethnopharmacology 163: 210-219. 
KUNWAR, R M; BURLAKOTI, C; CHOWDHARY, C L; BUSSMANN, R W (2010) Medicinal plants in far-west Nepal: Indigenous uses and pharmacological validity. Medicinal and Aromatic Plant Science and Biotechnology 4(1): 28-42.

LAMSAL, P; PANT, K P; KUMAR, L; ATREYA, K (2014) Diversity, uses, and threats in the Ghodaghodi Lake Complex, a Ramsar site in western lowland Nepal. ISRN Biodiversity, Article ID 680102. DOI:10.1155/2014/680102.

LAMSAL, P; PANT, K P; KUMAR, L; ATREYA, K (2014) Sustainable livelihoods through conservation of wetland resources: a case of economic benefits from Ghodaghodi Lake, western Nepal. Ecology and Society 20(1): 10. http://dx.doi. org/10.5751/ES-07172-200110.

LOWE, S; BROWNE, M; BOUDJELAS,S; DE POORTER,M (2000)100 of the world's worst invasive alien species: a selection from the global invasive species database (Vol. 12). Invasive Species Specialist Group, Auckland, New Zealand.

MANANDHAR, N P (2002) Plants and people of Nepal. Timber Press, USA.

MANDAL, S K; MUKHERJEE, A (2012) Diversity of Dicotyledonous plants in Wetlands of Puruliya District, West Bengal. In Maiti, G G; MukherjeE, S K (eds). Multidisciplinary Approaches in Angiosperm Systematics. University of Kalyani, Kalyani, WB, India; pp 403-9.

MoFE (2018a) National Ramsar Strategy and Action Plan, Nepal (2018-2024). Ministry of Forest and Environment, Government of Nepal, Kathmandu, Nepal..

MoFE (2018b) Integrated Lake Basin Management Plan of Lake Cluster of Pokhara Valley, Nepal (2018-2023). Ministry of Forests and Environment, Kathmandu, Nepal.

NIROULA, B; SINGH, K L B (2011) Aquatic plant resources of Betana wetland, Morang, Nepal. Our Nature 9(1): 146-155.

PRESS, J R; SHRESTHA, K K; SUTTON, D A (2000) Annotated checklist of the flowering plants of Nepal. Natural History Museum Publications, UK (updated online version: http://www.efloras.org/flora_page.aspx?flora_id=110, accessed date. 29 January 2021) 
RAMSAR CONVENTION SECRETARIAT (2013) The Ramsar Convention Manual: a guide to the Convention on Wetlands (Ramsar, Iran, 1971). $6^{\text {th }}$ eddition. Ramsar Convention Secretariat, Gland, Switzerland.

ROSKOV, Y; OWER, G.; ORRELL, T; NICOLSON, D; BAILLY, N; KIRK, P.M; BOURGOIN, T; DEWALT, R.E; DECOCK, W; NIEUKERKEN, E; PENEV, L; EDS. (2020) Species 2000 \& ITIS Catalogue of Life, 2020-09-01 Beta. Digital resource at www.catalogueoflife.org/col. Species 2000: Naturalis, Leiden, the Netherlands. ISSN 2405-8858. (accessed date: November 05, 2020).

SAH, J P; SINGH, R L; BHATTA, N (2002) Floristic diversity and use of plants in Ghodaghodi Lake Area, Nepal. Journal of Natural History Museum 21: 243-266.

SHARMA K; SAUD D S; JOSHI N (2019) Wetland flora of Rupandehi district, Nepal. Journal of Plant Resources 17(1): 58-68.

SHARMA, K; SAUD, D S; BHATTARAI,K R; DHAKAL, S; KHADKA, M K (2020) Wetland Plants and their Ethnobotanical Uses in Raja-Rani Tal, Letang, Morang, Nepal Journal of Plant Resources 18(1): 135-142.

SHRESTHA, B B; SHRESTHA, U B; SHARMA, K P; THAPA-PARAJULI, R B; DEVKOTA, A; SIWAKOTI, M (2019) Community perception and prioritization of invasive alien plants in Chitwan-Annapurna Landscape, Nepal. Journal of Environmental Management 229: 38-47.

SINGH, A; BALODI, K N; NAITHANI, S; SRIVASTAVA, A; SINGH, A; KWONNDUNG, E H (2017) Vascular plant diversity with special reference to the invasion of alien species on the Doon University Campus, Dehradun, India. International Journal of Biodiversity and Conservation 9(3): 56-76. DOI: $\underline{10.5897 / \mathrm{ijbc} 2016.1035}$

WATSON, M F; MKIYAMA, S; IKEDA, H; PENDRY, C; RAJBHANDARI, K R; SHRESTHA, K K eds. (2011) Flora of Nepal, Vol. 3. The Royal Botanic Garden Edinburgh, UK.

WU, Z; RAVEN, P H; HONG, D (1994-2008) Flora of China. Vol.4,8,11,13,14,16,18, 19,20,22,24,25. Science Press (Beijing) and Missouri Botanical Garden Press, St. Louis, USA. 
Pathak et al.: Floristic Diversity in the Lake Cluster of Pokhara Valley, Central Nepal

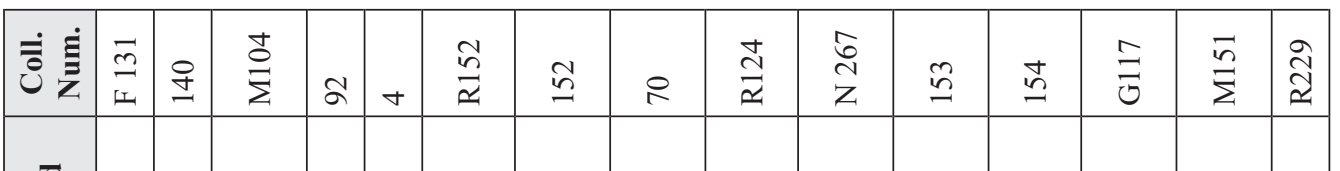

$\frac{3}{\sqrt{5}}$

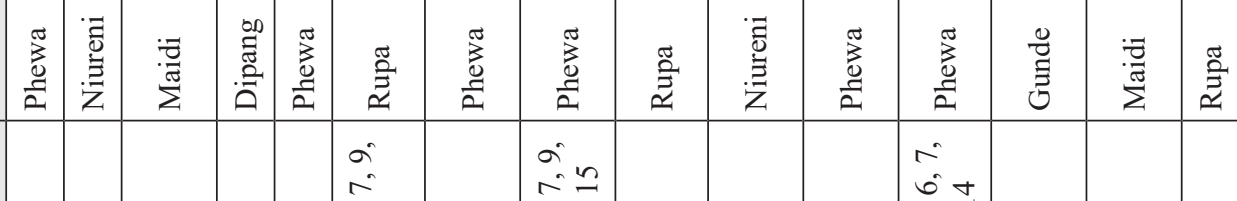

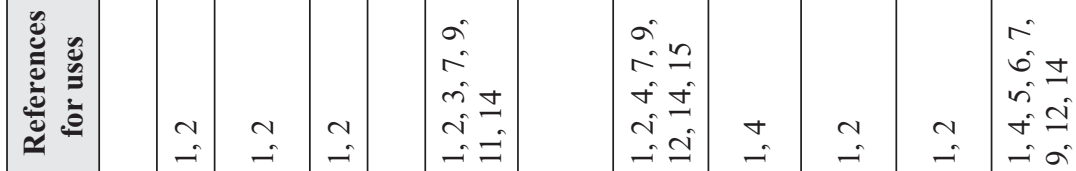

$\ddot{\infty}$

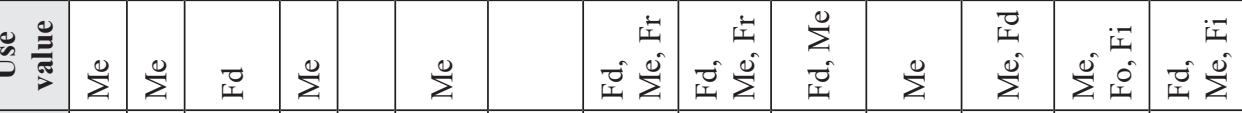

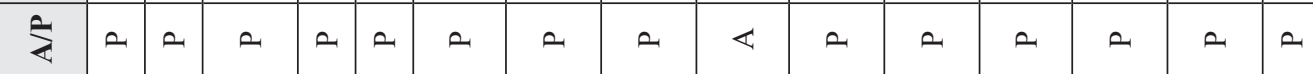

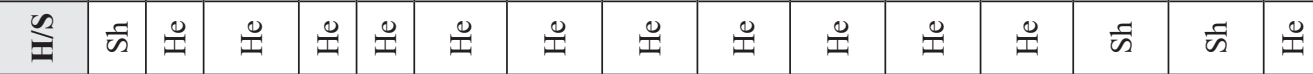

ฯ

$\frac{2}{\circlearrowright}$

\begin{tabular}{l|l|l|l|l|l|l|l|l|l|}
$\tilde{\frac{\pi}{z}}$ & - & - & - & - & - & - & - & - & -
\end{tabular}

\begin{tabular}{ll|l|l|l|l|l|l|l|l|l|l|l|l|l}
$\sum$ & 0 & 0 & 0 & 0 & 0 & $\Sigma$ & 0 & 0 & 0 & 0 & $\Sigma$ & 0 & 0 & 0 \\
\hline
\end{tabular}

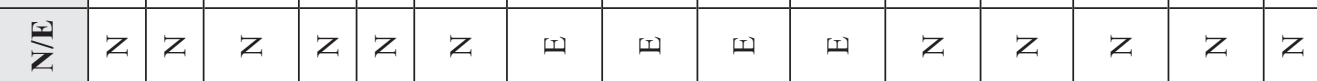

ํㅡㄹ

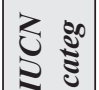

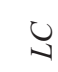

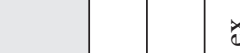

x.

$(2$

छ

\&

$\frac{\mathscr{d}}{0}$

Е

过

远

5
5
2
2
5

:

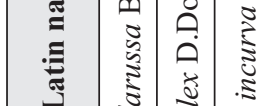

ฐิ

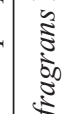

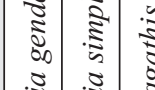

苟

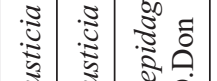

0
0
0
0
0

U

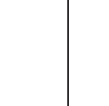

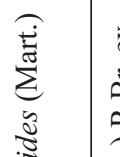

节

군

ป⿻

ह

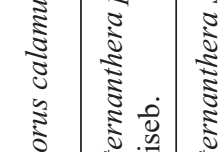

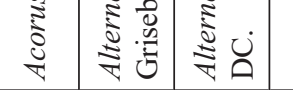

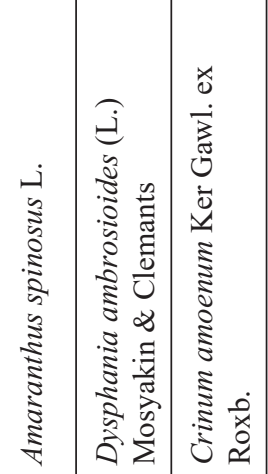

খ

খ

를

ن
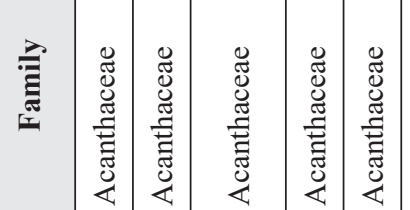

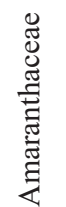

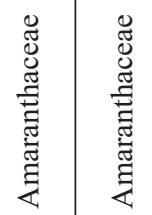

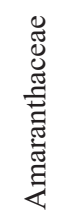

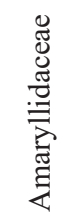

ᄅं

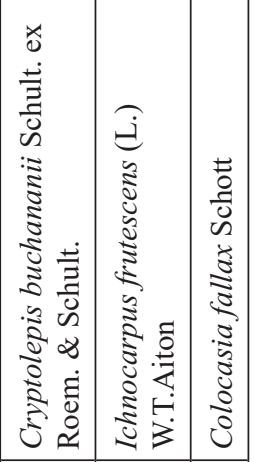

\begin{tabular}{l|l|l|l}
\hline Z & - & n & m
\end{tabular}

$\checkmark$

으

$=$ 


\begin{tabular}{|c|c|c|c|c|c|c|c|c|c|c|c|c|c|c|c|c|c|}
\hline$\stackrel{\Xi}{\Xi}$ & $\cong$ & $n$ & $\overline{\widetilde{Z}}$ & $\approx 2$ & $\stackrel{\ominus}{\Xi}$ & $\approx$ & $\underset{\Xi}{\beth}$ & $\stackrel{\check{\Xi}}{\underset{\Sigma}{\Sigma}}$ & $\frac{\hat{n}}{a}$ & $\stackrel{\infty}{\sigma}$ & $\frac{\hat{o}}{\Sigma}$ & $\frac{\infty}{n}$ & $\stackrel{\bullet}{\sim}$ & $\hat{0}$ & ڤે & $\underset{\sigma}{\mathbb{t}}$ & 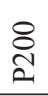 \\
\hline$\frac{\pi}{2}$ & 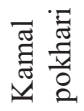 & $\frac{\sigma}{2}$ & $\stackrel{\widetilde{Z}}{\stackrel{\Xi}{\approx}}$ & 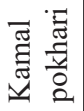 & $\sum_{\bar{\pi}}^{\bar{J}}$ & $\frac{\pi}{2}$ & 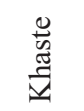 & $\frac{\pi}{0}$ & $\frac{\pi}{0}$ & 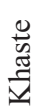 & $\sum_{\bar{\pi}}^{\bar{\pi}}$ & $\frac{\pi}{\frac{\pi}{2}}$ & 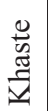 & 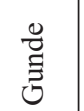 & $\begin{array}{c}\frac{\pi}{0} \\
\frac{0}{0} \\
\frac{\pi}{2}\end{array}$ & 离 & $\frac{\pi}{0}$ \\
\hline & & & $\begin{array}{l}= \\
i \\
i \\
i \\
i\end{array}$ & $\begin{array}{l}6 \\
+ \\
\therefore \simeq \\
-\infty \\
-\infty\end{array}$ & $\begin{array}{l}\nabla \\
i\end{array}$ & $\begin{array}{l}\cong \\
-\end{array}$ & $\begin{array}{l}n \\
\stackrel{n}{n} \\
=\end{array}$ & $N$ & $\cong$ & & - & 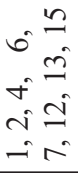 & $\begin{array}{l}\simeq \\
\approx \\
\therefore \\
\therefore \\
\therefore\end{array}$ & $\begin{array}{l}\sim \\
\sim\end{array}$ & & & $\stackrel{n}{n}$ \\
\hline & & & $\sum^{0}$ & $\begin{array}{l}\dot{1} \\
\sum^{0}\end{array}$ & $\sum^{\infty}$ & $\sum_{i=1}^{0}$ & 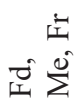 & $\sum_{\Sigma}^{0}$ & $\sum_{\Sigma}^{0}$ & $\sum_{\Sigma}^{0}$ & $\sum^{0}$ & 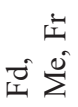 & $\begin{array}{l}\Gamma^{\pi} \\
\sum^{\infty}\end{array}$ & 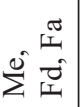 & & & 它 \\
\hline a & $\ll$ & $a$ & $a$ & $\varangle$ & $\ll$ & $\varangle$ & $\ll$ & $a$ & $\ll$ & $\varangle$ & $a$ & $\ll$ & $a$ & $\ll$ & $\ll$ & $\ll$ & $a$ \\
\hline$\stackrel{\mathscr{I}}{ \pm}$ & I & $\stackrel{0}{I}$ & $\stackrel{\oplus}{I}$ & $\stackrel{0}{I}$ & $\stackrel{\ddot{I}}{\ddot{I}}$ & $\stackrel{0}{\dddot{I}}$ & $\stackrel{0}{I}$ & $\bar{\sim}$ & $\stackrel{0}{I}$ & $\stackrel{\mathscr{I}}{\stackrel{1}{2}}$ & $\overline{\tilde{n}}$ & $\stackrel{0}{I}$ & $\stackrel{0}{\Perp}$ & $\stackrel{0}{I}$ & 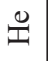 & $\stackrel{\ddot{I}}{\ddot{I}}$ & $\stackrel{0}{\mathbb{I}}$ \\
\hline تَ & 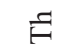 & $\stackrel{0}{ \pm}$ & 己ี & 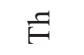 & F & $\Xi$ & $F$ & లี & 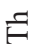 & $\tilde{F}$ & छี & 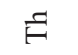 & $\stackrel{\mathscr{I}}{\stackrel{0}{*}}$ & $\Xi$ & 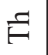 & $\Xi$ & 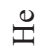 \\
\hline$N$ & - & - & - & - & - & & - & - & - & - & - & - & - & - & - & - & - \\
\hline$\Sigma$ & D & D & D & ص & ค & ค & ค & ص & D & D & D & D & ค & ค & ค & ค & D \\
\hline 디 & Z & Z & 디 & [工] & 디 & Z & 口I & [工] & 디 & z & z & 디 & Z & Z & 디 & Z & 디 \\
\hline 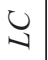 & $\underset{\exists}{U}$ & & & & & & & & & & & $\underset{G}{U}$ & & & & & \\
\hline
\end{tabular}

\begin{tabular}{|c|c|c|c|c|c|c|c|c|c|c|c|c|c|c|c|c|c|}
\hline & & & & & & & & & & & & & & & & & \\
\hline 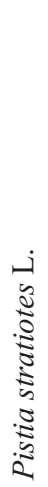 & 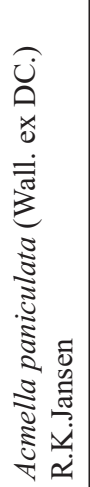 & 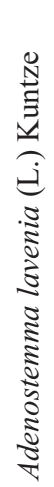 & 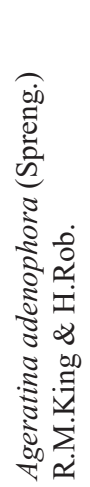 & 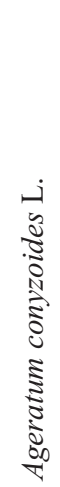 & 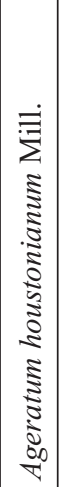 & 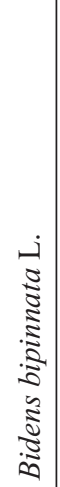 & 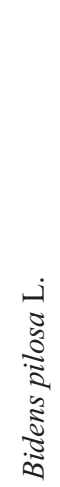 & 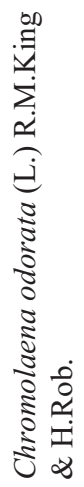 & 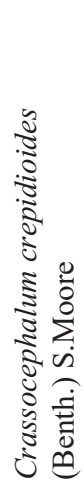 & 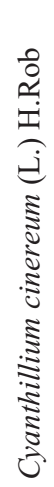 & 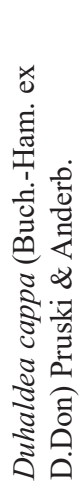 & 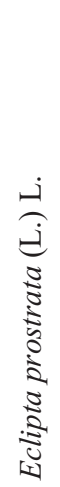 & 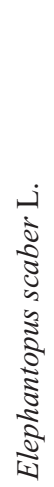 & 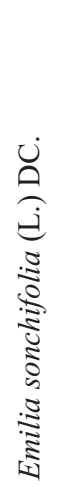 & 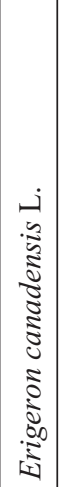 & 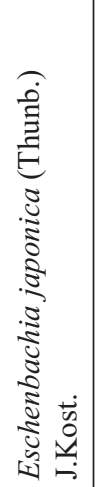 & 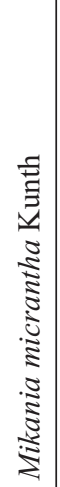 \\
\hline 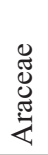 & 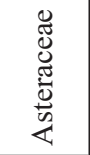 & 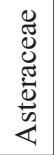 & 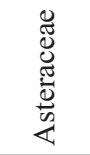 & 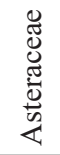 & 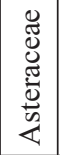 & 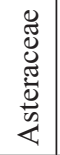 & 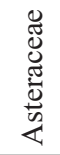 & 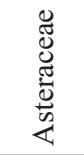 & 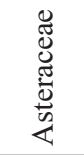 & 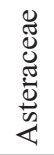 & 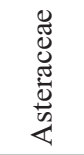 & 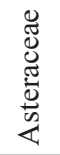 & 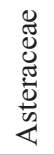 & 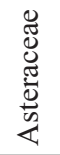 & 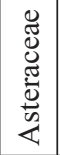 & 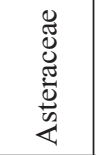 & 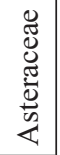 \\
\hline$\underset{-}{0}$ & $\beth$ & $\infty$ & $\stackrel{2}{2}$ & ิㅗ & $\vec{\sim}$ & ก & 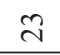 & $\stackrel{\sim}{\sim}$ & $\approx$ & $\stackrel{\circ}{\sim}$ & $\hat{\sim}$ & $\stackrel{\infty}{\sim}$ & ते & లి & $\vec{m}$ & లె & $m$ \\
\hline
\end{tabular}


20 Pathak et al.: Floristic Diversity in the Lake Cluster of Pokhara Valley, Central Nepal

\begin{tabular}{|c|c|c|c|c|c|c|c|c|c|c|c|c|c|c|c|c|c|c|}
\hline$\stackrel{+}{\Delta}$ & $\frac{\sqrt{7}}{\gamma}$ & $\bar{\infty}$ & 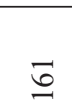 & $\frac{\widetilde{O}}{2}$ & $\frac{\Delta}{0}$ & $\underset{⿱ 亠}{\stackrel{\infty}{N}}$ & $\underset{\sim}{\stackrel{\Xi}{\simeq}}$ & ్ㅡ & $\begin{array}{l}\vec{\sim} \\
\infty\end{array}$ & $\stackrel{n}{\varrho}$ & $\hat{\sim}$ & $\hat{\sigma}$ & $\stackrel{n}{\underline{n}}$ & $\underset{0}{0}$ & in & 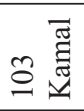 & $\stackrel{\text { m}}{\bar{z}}$ & $\ddot{\imath}$ \\
\hline$\frac{\pi}{0}$ & 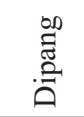 & 莺 & $\frac{\pi}{0}$ & $\frac{\pi}{0}$ & $\frac{\pi}{0}$ & 㽕 & 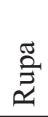 & $\frac{\pi}{0}$ & 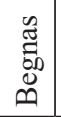 & 胥 & 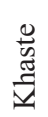 & 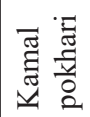 & $\stackrel{\widetilde{a}}{\ddot{2}}$ & 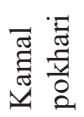 & $\frac{\pi}{0}$ & 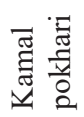 & 离 & $\underset{\Xi}{\Xi}$ \\
\hline & & $\stackrel{\sim}{\sim}$ & $\stackrel{\sim}{\sim}$ & - & $\stackrel{\sim}{\sim}$ & $\stackrel{\sim}{\sim}$ & $\stackrel{a}{\rightarrow}$ & - & $\begin{array}{l}m \\
m \\
i \\
-\end{array}$ & & & - & $\stackrel{\sim}{\leftrightarrows}$ & $\stackrel{\sim}{\leadsto}$ & & $\stackrel{\sim}{\sim}$ & & 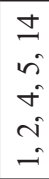 \\
\hline & & $\begin{array}{l}\overrightarrow{1} \\
\sum^{0}\end{array}$ & 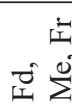 & $\vec{I}$ & $\sum^{\infty}$ & $\sum_{\Sigma}^{0}$ & $\sum^{0}$ & $\overrightarrow{|c|}$ & $\begin{array}{l}\vec{\alpha} \\
\dot{\Sigma}\end{array}$ & & & $\vec{I}$ & $\sum_{i=1}^{0}$ & $\sum_{i=1}^{0}$ & & $\sum_{i=1}^{0}$ & & $\begin{array}{l}\text { it } \\
\dot{e}\end{array}$ \\
\hline$\varangle$ & $\varangle$ & $\varangle$ & $\varangle$ & $\varangle$ & $\varangle$ & $\varangle$ & $\varangle$ & $\varangle$ & $a$ & $a$ & $\varangle$ & $a$ & $\varangle$ & $a$ & $a$ & $a$ & $a$ & 2 \\
\hline$\stackrel{0}{1}$ & $\stackrel{0}{I}$ & $\stackrel{\oplus}{I}$ & $\stackrel{0}{I}$ & 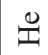 & $\stackrel{\mathscr{I}}{1}$ & $\stackrel{0}{ \pm}$ & $\stackrel{\oplus}{I}$ & $\stackrel{\oplus}{\Psi}$ & $\exists$ & $\bar{\sim}$ & $\stackrel{0}{I}$ & $\stackrel{\mathscr{I}}{1}$ & $\stackrel{\oplus}{\Psi}$ & $\stackrel{0}{\stackrel{I}{*}}$ & $\stackrel{\mathscr{I}}{1}$ & $\stackrel{\mathscr{I}}{ }$ & $\stackrel{\oplus}{1}$ & $\stackrel{\oplus}{\Psi}$ \\
\hline$\Xi$ & $\Xi$ & $\Xi$ & 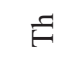 & $\Xi$ & 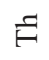 & $\Xi$ & $\cong$ & $\Xi$ & $\bar{n}$ & $\stackrel{0}{I}$ & $\Xi$ & تَ & 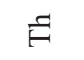 & $\stackrel{0}{I}$ & $\dot{U}$ & Ü & $\dot{U}$ & U \\
\hline- & - & - & - & - & - & - & - & - & - & - & - & - & - & - & - & - & - & - \\
\hline D & D & D & D & O & 0 & D & D & 0 & 0 & D & D & D & 0 & D & D & $\Sigma$ & $\Sigma$ & $\Sigma$ \\
\hline 디 & Z & 디 & Z & 디 & 디 & 디 & 디 & Z & $\mathrm{Z}$ & Z & Z & 디 & 山 & Z & Z & Z & Z & z \\
\hline 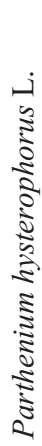 & 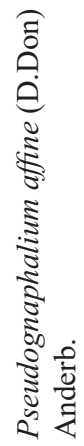 & 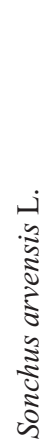 & 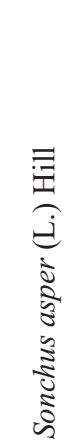 & 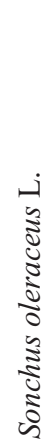 & 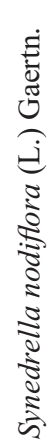 & 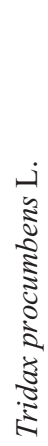 & 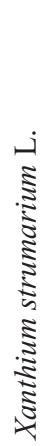 & 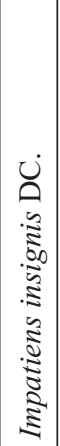 & 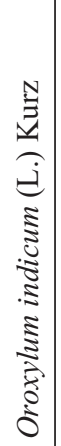 & 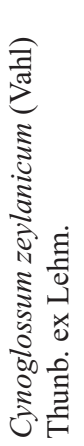 & 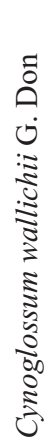 & 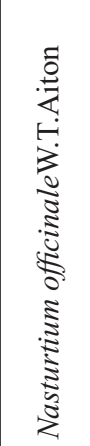 & 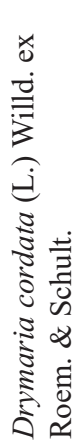 & 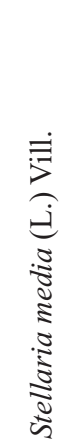 & 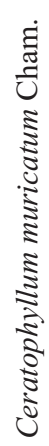 & 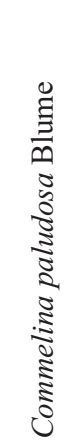 & 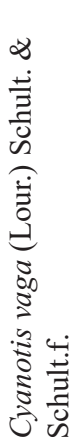 & 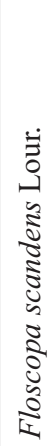 \\
\hline 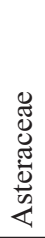 & 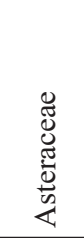 & 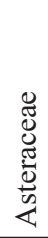 & 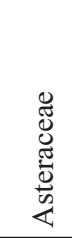 & 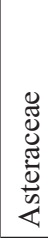 & 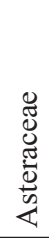 & 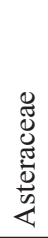 & 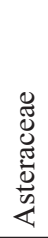 & 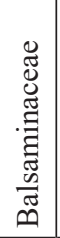 & 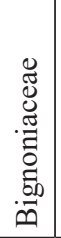 & 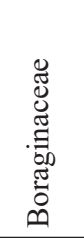 & 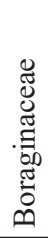 & 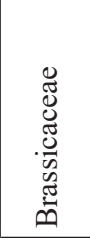 & 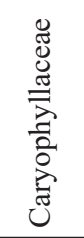 & 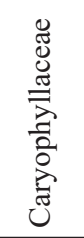 & 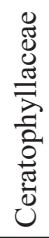 & 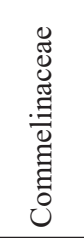 & 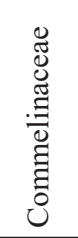 & 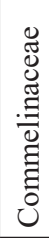 \\
\hline 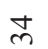 & $m$ & r & $\hat{n}$ & $\stackrel{\infty}{m}$ & సે & \& & $\vec{\gamma}$ & $\stackrel{\sim}{\mathcal{F}}$ & $\mathscr{\vartheta}$ & 寸 & $\stackrel{\wp}{f}$ & fo & F & $\stackrel{\infty}{+}$ & $\stackrel{\vartheta}{q}$ & in & $\bar{n}$ & $\tilde{N}$ \\
\hline
\end{tabular}




\begin{tabular}{|c|c|c|c|c|c|c|c|c|c|c|c|c|c|c|c|c|c|}
\hline$\stackrel{?}{\stackrel{9}{a}}$ & $\bar{\sigma}$ & $\underset{0}{\infty}$ & $\stackrel{\infty}{\stackrel{\infty}{\simeq}}$ & $\frac{8}{\underline{1}}$ & $r$ & oิ & $\frac{\bar{n}}{a}$ & 字 & 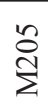 & $\vec{\sim}$ & $\underset{\Xi}{\tilde{a}}$ & $\frac{\Xi}{\Sigma}$ & $\stackrel{\sim}{\sim}$ & $\infty$ & $\stackrel{0}{\Xi}$ & $0 \frac{0}{0}$ & $\underset{\vec{a}}{\overrightarrow{1}}$ \\
\hline 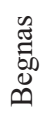 & $\frac{\pi}{0}$ & 总 & 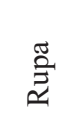 & 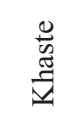 & $\stackrel{\widetilde{a}}{\stackrel{\Xi}{2}}$ & $\frac{\pi}{0}$ & $\frac{\tilde{3}}{2}$ & $\frac{\pi}{2}$ & $\sum^{-\bar{J}}$ & $\frac{\pi}{2}$ & $\frac{\pi}{0}$ & $\sum_{i}^{\cdot \bar{\pi}}$ & 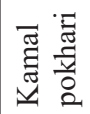 & 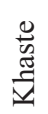 & 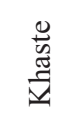 & $\frac{\pi}{2}$ & 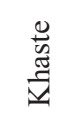 \\
\hline $\begin{array}{l}\sim \\
a \\
\therefore \\
\therefore\end{array}$ & $\begin{array}{l} \pm \\
\stackrel{-}{2} \\
\therefore \\
\stackrel{-}{-}\end{array}$ & & $\stackrel{n}{i}$ & $\stackrel{\sim}{\sim}$ & $N$ & & & $\frac{\sim}{a}$ & $\begin{array}{l} \pm \\
\stackrel{\sim}{\sim} \\
\text { i }\end{array}$ & & $\stackrel{\sim}{\simeq}$ & $\begin{array}{l}\simeq \\
a \\
i\end{array}$ & & & 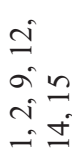 & $\stackrel{+}{\sim}$ & \\
\hline$\sum_{i}^{0}$ & $\stackrel{\oplus}{ \pm} \Sigma^{0}$ & & $\sum_{i=1}^{0}$ & 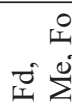 & $\begin{array}{l}\dot{1} \\
\sum^{0}\end{array}$ & & & $\dot{15}$ & $\begin{array}{l}\dot{15} \\
\dot{0}\end{array}$ & & $\stackrel{0}{\Sigma}$ & $\sum_{i=1}^{0}$ & & & $\sum_{\substack{0 \\
0}}^{0}$ & $\begin{array}{l}0 \\
\text { i } \\
\text { it }\end{array}$ & \\
\hline$\varangle$ & $a$ & $\varangle$ & $a$ & $a$ & $a$ & $a$ & $\varangle$ & $a$ & $\varangle$ & $\varangle$ & 2 & $\varangle$ & $\varangle$ & $\varangle$ & $a$ & $a$ & $\varangle$ \\
\hline$\stackrel{0}{1}$ & $\stackrel{0}{1}$ & $\stackrel{0}{1}$ & $\stackrel{0}{1}$ & $\stackrel{0}{I}$ & $\stackrel{0}{1}$ & $\stackrel{0}{I}$ & $\stackrel{0}{I}$ & $\stackrel{0}{1}$ & $\stackrel{0}{1}$ & $\stackrel{0}{1}$ & $\stackrel{0}{\dddot{I}}$ & $\stackrel{0}{I}$ & 章 & $\stackrel{0}{1}$ & $\stackrel{0}{1}$ & $\stackrel{0}{1}$ & $\stackrel{0}{I}$ \\
\hline$\cong$ & లี & 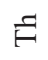 & $\stackrel{\ddot{I}}{1}$ & $\stackrel{0}{1}$ & $\dot{U}$ & ت̇ & $\stackrel{F}{F}$ & ت̇ & $\Xi$ & $\Xi$ & ث̈ & $\Xi$ & 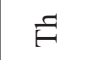 & $\cong$ & Ü & $\dot{U}$ & $\Xi$ \\
\hline- & - & - & - & - & - & - & - & - & - & - & - & - & - & - & - & - & - \\
\hline$\Sigma$ & D & D & $\Sigma$ & D & $\Sigma$ & $\Sigma$ & $\Sigma$ & $\Sigma$ & $\Sigma$ & $\Sigma$ & $\Sigma$ & $\Sigma$ & $\Sigma$ & $\Sigma$ & $\Sigma$ & $\Sigma$ & $\Sigma$ \\
\hline Z & 디 & 디 & Z & Z & Z & Z & Z & Z & Z & Z & Z & Z & Z & Z & Z & Z & Z \\
\hline & & & 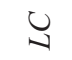 & & U & & & $\underset{৩}{\cup}$ & U & & $\stackrel{\ddots}{\ddots}$ & $\underset{\exists}{U}$ & & U & $U$ & & \\
\hline 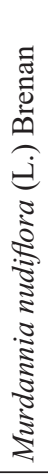 & 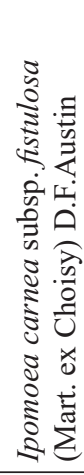 & 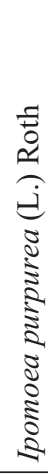 & 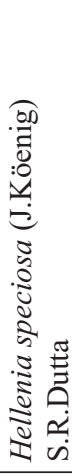 & 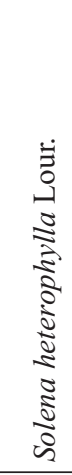 & 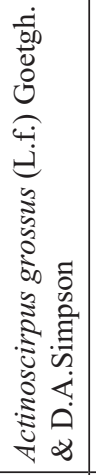 & 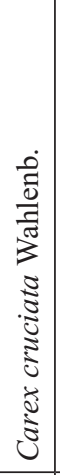 & 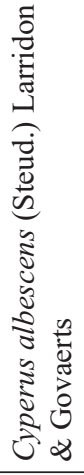 & 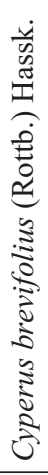 & 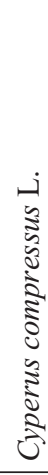 & 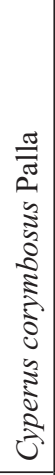 & 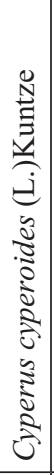 & 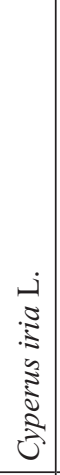 & 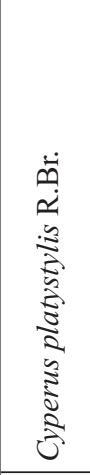 & 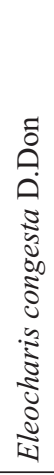 & 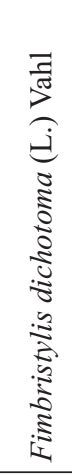 & 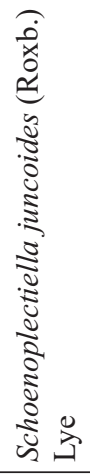 & 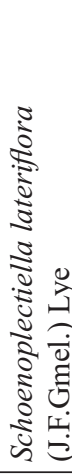 \\
\hline 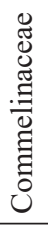 & 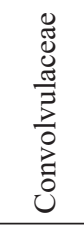 & 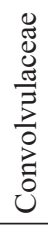 & 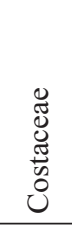 & 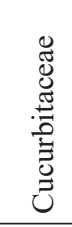 & 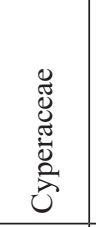 & 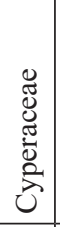 & 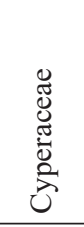 & 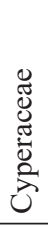 & 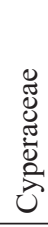 & 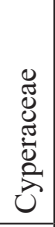 & 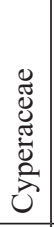 & 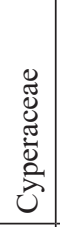 & 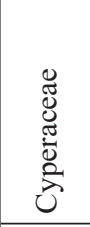 & 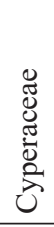 & 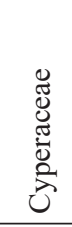 & 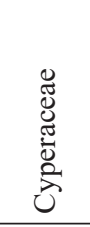 & 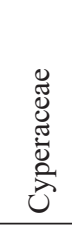 \\
\hline$n$ & 芯 & $n$ & in & in & $\stackrel{\infty}{n}$ & in & 8 & $\bar{\sigma}$ & $\widetilde{\sigma}$ & $\hat{6}$ & ț & 6 & 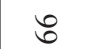 & $\hat{6}$ & $\infty$ & g) & R \\
\hline
\end{tabular}


22 Pathak et al.: Floristic Diversity in the Lake Cluster of Pokhara Valley, Central Nepal

\begin{tabular}{|c|c|c|c|c|c|c|c|c|c|c|c|c|c|c|c|c|c|}
\hline$\stackrel{\infty}{\check{a}}$ & 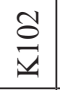 & $\begin{array}{l}\stackrel{\circ}{0} \\
\vec{\Delta}\end{array}$ & $\frac{\tilde{O}}{\widetilde{\alpha}}$ & $\underset{-}{\infty}$ & $\bar{a}$ & $\begin{array}{l}\text { ָ̇ } \\
\text { ¿ }\end{array}$ & $\stackrel{\stackrel{ \pm}{J}}{\stackrel{\Sigma}{\Sigma}}$ & $\cong$ & 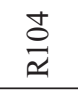 & $\underset{\sim}{\stackrel{\sim}{*}}$ & $\underset{-}{+}$ & o̊ & $\begin{array}{l}\overrightarrow{0} \\
\simeq 2\end{array}$ & $\vec{\gamma}$ & $\stackrel{\circ}{+}$ & $\hat{\infty}$ & 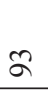 \\
\hline$\stackrel{\widetilde{2}}{\stackrel{\Xi}{\approx}}$ & 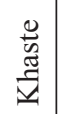 & 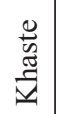 & 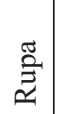 & 司 & $\sum_{\bar{\pi}}^{\bar{\pi}}$ & $\frac{\pi}{2}$ & $\sum_{\bar{\pi}}^{\pi}$ & $\frac{\pi}{0}$ & 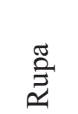 & 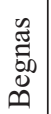 & $\frac{\pi}{3}$ & $\begin{array}{l}\stackrel{8}{E} \\
\Xi \\
0\end{array}$ & $\stackrel{\widetilde{ٌ}}{\Xi}$ & $\frac{\pi}{0}$ & $\frac{\pi}{2}$ & 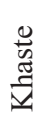 & 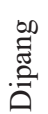 \\
\hline $\begin{array}{l} \pm \\
\stackrel{5}{\leftrightarrows}\end{array}$ & $\stackrel{\sim}{\curvearrowleft}$ & & $\stackrel{\sim}{\because}$ & & $\begin{array}{l}m \\
i \\
i\end{array}$ & - & & $N$ & - & $\stackrel{\sim}{-}$ & $\stackrel{\sim}{\sim}$ & $N$ & $\stackrel{\sim}{-}$ & & & - & \\
\hline $\begin{array}{l}0 \\
\stackrel{0}{\mid c}\end{array}$ & O & & $\sum_{i=1}^{0}$ & & 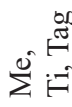 & $\sum_{\Sigma}^{0}$ & & $\sum^{0}$ & $\begin{array}{l}\dot{0} \\
\dot{0}\end{array}$ & $\begin{array}{l}\text { के } \\
\sum^{0}\end{array}$ & $\begin{array}{l}0 \\
\stackrel{1}{\infty} \\
e^{0}\end{array}$ & $\sum^{0}$ & 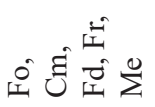 & & & $\stackrel{\Sigma}{\Sigma}^{0}$ & \\
\hline$a$ & $a$ & $a$ & $a$ & $a$ & $a$ & $\varangle$ & $a$ & $\ll$ & $a$ & $a$ & $a$ & $a$ & $a$ & $a$ & $a$ & $a$ & $a$ \\
\hline$\stackrel{\mathscr{I}}{1}$ & $\stackrel{\mathscr{I}}{1}$ & $\stackrel{0}{ \pm}$ & $\stackrel{0}{\mathbb{I}}$ & $\stackrel{0}{I}$ & 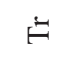 & $\stackrel{0}{I}$ & $\overline{\mathfrak{n}}$ & $\stackrel{0}{ \pm}$ & है & $\dot{F}$ & $\exists$ & שี & $\dot{F}$ & $\stackrel{0}{I}$ & $\stackrel{0}{I}$ & שี & $\bar{n}$ \\
\hline U゙ & تَن & تَن & $\stackrel{\oplus}{I}$ & $\stackrel{\mathscr{I}}{ }$ & $\bar{\varepsilon}$ & $E$ & $\tilde{U}$ & $\Xi$ & சี & $\bar{a}$ & $\bar{a}$ & $\stackrel{0}{I}$ & $\bar{q}$ & $\stackrel{\mathscr{I}}{\stackrel{0}{*}}$ & $\stackrel{0}{I}$ & $\stackrel{0}{I}$ & $\stackrel{0}{I}$ \\
\hline- & - & - & - & - & - & - & - & - & - & - & - & - & - & - & - & - & - \\
\hline$\Sigma$ & $\Sigma$ & $\Sigma$ & D & D & D & $\Sigma$ & D & D & a & a & D & D & D & a & a & D & D \\
\hline Z & Z & Z & Z & Z & Z & Z & Z & Z & Z & Z & Z & Z & Z & Z & 디 & Z & Z \\
\hline$\underset{J}{U}$ & $\underset{\exists}{U}$ & & & & $\underset{V}{U}$ & $\underset{\exists}{U}$ & & & $\underset{J}{U}$ & & $\underset{\forall}{U}$ & $\underset{\checkmark}{\cup}$ & $\stackrel{\cup}{\cup}$ & & & & \\
\hline 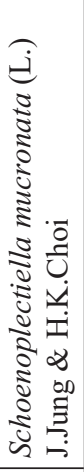 & 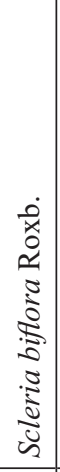 & 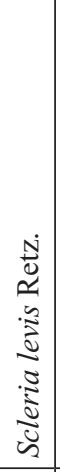 & 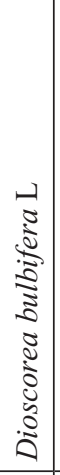 & 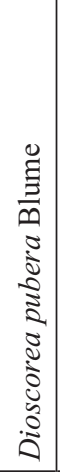 & 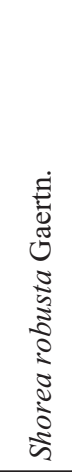 & 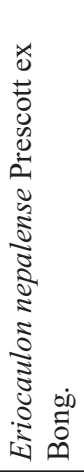 & 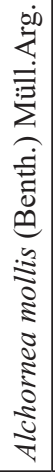 & 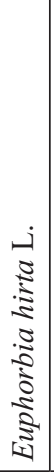 & 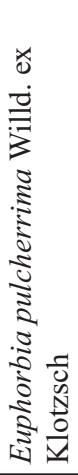 & 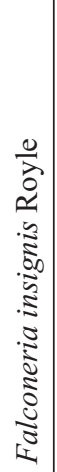 & 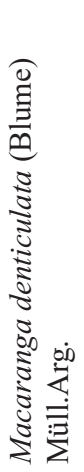 & 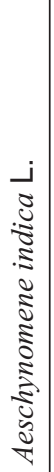 & 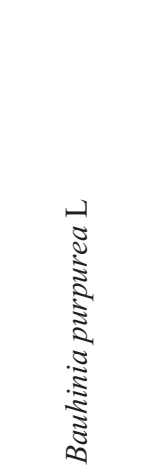 & 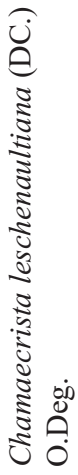 & 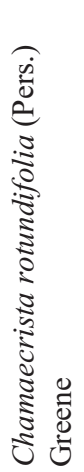 & 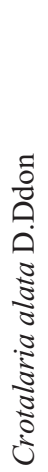 & 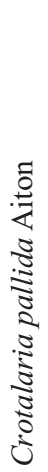 \\
\hline 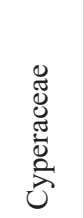 & 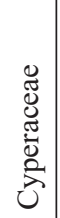 & 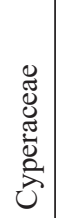 & 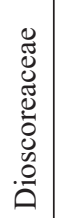 & 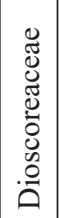 & 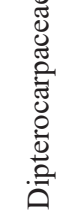 & 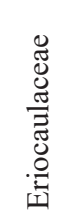 & 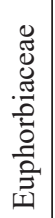 & 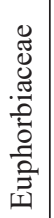 & 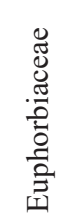 & 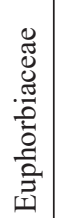 & 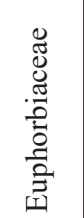 & 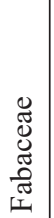 & 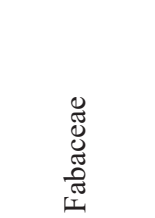 & 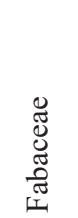 & 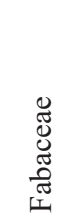 & 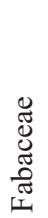 & 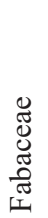 \\
\hline$\nabla$ & $\mathbb{N}$ & $\Re$ & $\underset{d}{ }$ & $\stackrel{n}{\sim}$ & 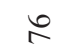 & $\therefore$ & $\stackrel{\infty}{\sim}$ & 2 & $\infty$ & $\vec{\infty}$ & ๙ & $\tilde{\infty}$ & ఫ & $\ddot{\infty}$ & $\infty$ & $\hat{\infty}$ & $\infty$ \\
\hline
\end{tabular}




\begin{tabular}{|c|c|c|c|c|c|c|c|c|c|c|c|c|c|c|c|c|}
\hline 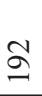 & $\vec{a}$ & in & $\stackrel{ \pm}{\sigma}$ & $\hat{a}$ & 尺े & $\bar{m}$ & 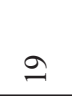 & $\stackrel{ }{\stackrel{n}{1}}$ & N & $\frac{n}{\Sigma}$ & ㄱำ & 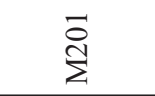 & ષ્ત & 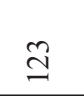 & Z & $\bar{\lambda}$ \\
\hline 㽕 & $\frac{\pi}{2}$ & $\frac{\pi}{2}$ & $\frac{\pi}{2}$ & 蔦 & $\sum_{\bar{\pi}}^{\cdot \bar{\pi}}$ & $\stackrel{\widetilde{a}}{\stackrel{\Xi}{\approx}}$ & $\frac{\pi}{2}$ & $\frac{\pi}{2}$ & $\sum_{\frac{1}{2}}^{\pi}$ & $\frac{\pi}{2}$ & $\frac{\pi}{2}$ & : & $\stackrel{\widetilde{a}}{\overrightarrow{2}}$ & $\begin{array}{l}\cdot \overline{0} \\
\stackrel{0}{\Xi} \\
\dot{Z}\end{array}$ & 离 & 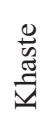 \\
\hline$\stackrel{\sim}{\sim}$ & $\stackrel{\sim}{\sim}$ & $\stackrel{\sim}{\sim}$ & $\stackrel{\sim}{-}$ & $\stackrel{\sim}{\sim}$ & & & 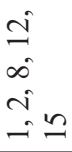 & $\stackrel{\sim}{-}$ & $\stackrel{\sim}{\sim}$ & $\begin{array}{l}\sim \\
\infty \\
\stackrel{-}{-} \\
\stackrel{-}{0}\end{array}$ & & $\because$ & $\stackrel{\sim}{\because}$ & $\stackrel{\sim}{\cong}$ & $\begin{array}{l}0 \\
+ \\
i \\
i\end{array}$ & $\stackrel{\sim}{\sim}$ \\
\hline$\sum^{0}$ & $\sum^{0}$ & $\sum^{0}$ & i & $\sum^{0}$ & & & $\sum^{0}$ & $\sum^{0}$ & $\begin{array}{l}\sum_{\Sigma}^{0} \\
\tilde{E} \\
\dot{1}\end{array}$ & $\begin{array}{l}\sum_{i}^{0} \\
\tilde{D}= \\
\end{array}$ & & $\sum^{0} \overrightarrow{|c|}$ & 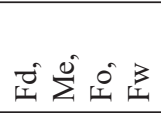 & $\sum^{0}$ & 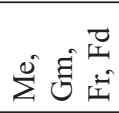 & $\stackrel{0}{\Sigma}$ \\
\hline$a$ & $a$ & $\varangle$ & $\varangle$ & $a$ & $\varangle$ & $a$ & $a$ & $a$ & $\varangle$ & $\varangle$ & $\varangle$ & $a$ & $a$ & $a$ & $a$ & 0 \\
\hline చే & 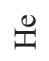 & $\stackrel{0}{\mathbb{I}}$ & $\stackrel{0}{1}$ & $\stackrel{0}{I}$ & $\stackrel{0}{I}$ & $\bar{n}$ & $\stackrel{\mathscr{I}}{1}$ & च & $\bar{n}$ & $\bar{\varpi}$ & $\stackrel{0}{\Psi}$ & 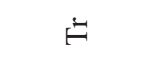 & $\dot{H}$ & $\stackrel{0}{\underline{I}}$ & $\stackrel{\oplus}{\mathbb{1}}$ & $\stackrel{\oplus}{I}$ \\
\hline 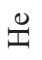 & $\stackrel{\mathscr{I}}{I}$ & $\Xi$ & $\Xi$ & Eี & $\Xi$ & $\bar{a}$ & $\stackrel{0}{I}$ & 己ี & $\Xi$ & 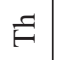 & 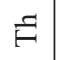 & $\bar{Q}$ & $\bar{a}$ & $\bar{Q}$ & $\dot{U}$ & $\stackrel{\mathscr{I}}{ }$ \\
\hline- & - & - & - & - & - & $m$ & - & - & - & - & - & - & - & - & - & - \\
\hline D & 0 & D & D & D & D & D & D & D & 0 & 0 & D & D & 0 & D & $\Sigma$ & D \\
\hline z & Z & Z & Z & Z & z & Z & 디 & Z & 띠 & 山 & 디 & Z & Z & 디 & Z & Z \\
\hline & & & & & & & U & & & & & $\underset{\exists}{U}$ & $\underset{J}{U}$ & & U & \\
\hline 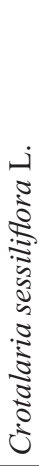 & 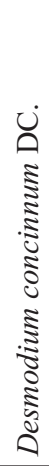 & 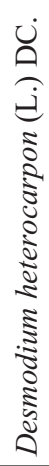 & 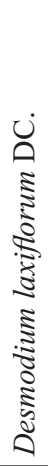 & 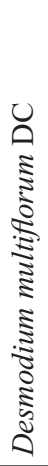 & 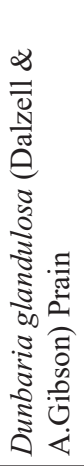 & 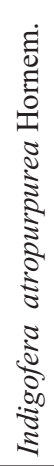 & 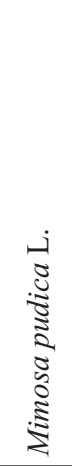 & 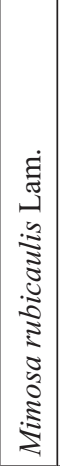 & 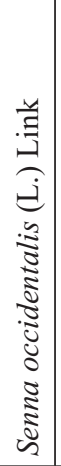 & 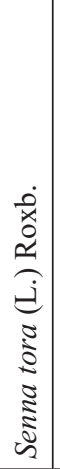 & 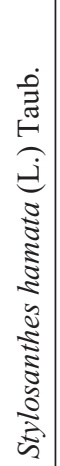 & 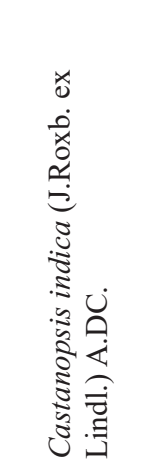 & 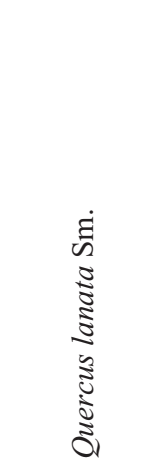 & 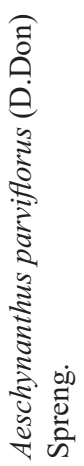 & 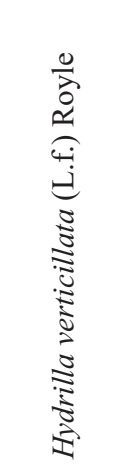 & 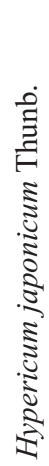 \\
\hline 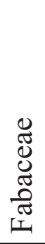 & $\begin{array}{l}\mathscr{J} \\
\tilde{J} \\
\tilde{\Xi} \\
\tilde{\pi} \\
\tilde{T}\end{array}$ & 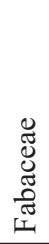 & 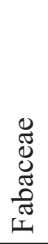 & 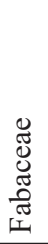 & 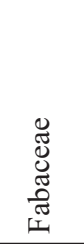 & 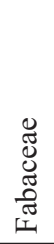 & 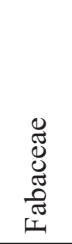 & 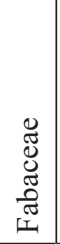 & 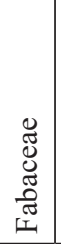 & 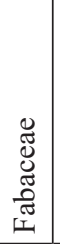 & 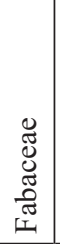 & 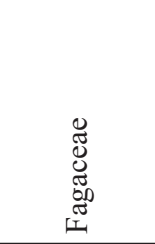 & 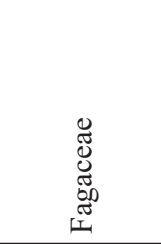 & 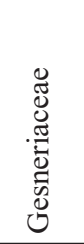 & 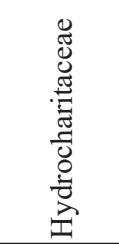 & 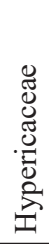 \\
\hline ळे & \& & $\bar{a}$ & ส & 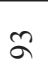 & J & 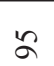 & ๙ & sa & $\stackrel{\infty}{\circ}$ & হ & 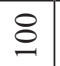 & $\overline{0}$ & ธิ & $\tilde{o}$ & $\stackrel{\Xi}{O}$ & $\stackrel{2}{0}$ \\
\hline
\end{tabular}


24 Pathak et al.: Floristic Diversity in the Lake Cluster of Pokhara Valley, Central Nepal

\begin{tabular}{|c|c|c|c|c|c|c|c|c|c|c|c|c|c|c|c|c|c|c|}
\hline 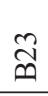 & ஓे & $\frac{\sim}{\widetilde{\sigma}}$ & $\frac{\vec{\beth}}{\underline{\simeq}}$ & $\hat{\sim}$ & రิ & $\stackrel{0}{0}$ & $\stackrel{\sim}{\sim}$ & $\stackrel{\Sigma}{\bar{z}}$ & $\underset{\mathbb{Z}}{\stackrel{\Xi}{\Delta}}$ & $\infty$ & $\underset{\sim}{\infty}$ & $\stackrel{2}{2}$ & $\stackrel{⿱}{\sim}$ & $\frac{2}{2}$ & $\overline{\widetilde{\beth}}$ & $\sqrt[\vec{n}]{\sim}$ & $\begin{array}{l}0 \\
\stackrel{2}{\underline{V}}\end{array}$ & $\begin{array}{l}\infty \\
\sim\end{array}$ \\
\hline 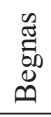 & $\sum_{\bar{\pi}}^{\bar{\pi}}$ & 莺 & 芯 & $\frac{\pi}{2}$ & 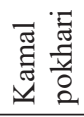 & $\frac{\pi}{2}$ & $\stackrel{\widetilde{2}}{\vec{\approx}}$ & 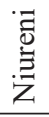 & 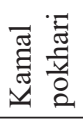 & $i_{\bar{\pi}}^{\bar{\pi}}$ & 咅 & 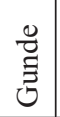 & $\begin{array}{c}\stackrel{\tilde{v}}{E} \\
\Xi \\
\Xi\end{array}$ & 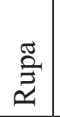 & 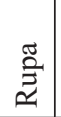 & $\sum_{\bar{\pi}}^{\frac{\pi}{\pi}}$ & 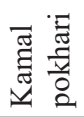 & 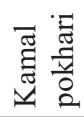 \\
\hline- & 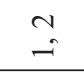 & & & $\stackrel{\sim}{-}$ & $\hat{a}$ & $N$ & $N$ & $N$ & $\stackrel{\sim}{\leadsto}$ & $\stackrel{\sim}{\cong}$ & $\stackrel{\sim}{\cong}$ & & $\stackrel{\sim}{\sim}$ & & $\stackrel{\sim}{\curvearrowleft}$ & & $\stackrel{\sim}{\sim}$ & $N$ \\
\hline$\stackrel{0}{\Sigma}$ & $\begin{array}{l}0 \\
\dot{I}^{\circ} \\
\dot{s}^{\circ}\end{array}$ & & & $\stackrel{\infty}{\Sigma}$ & $\stackrel{0}{\Sigma}$ & $\stackrel{n}{\Sigma}$ & $\stackrel{\infty}{\Sigma}$ & $\stackrel{n}{\Sigma}$ & $\begin{array}{l}\overrightarrow{\underline{a}} \\
\vec{\Sigma}\end{array}$ & $\sum_{\vec{I}}^{0}$ & $\stackrel{\Sigma}{\Sigma}^{\infty}$ & & $\stackrel{\infty}{\Sigma}$ & & $\begin{array}{l}0 \\
\sum^{0} \\
0 \\
0\end{array}$ & & $\stackrel{n}{\Sigma}$ & $\stackrel{0}{\Sigma}$ \\
\hline a & $a$ & $a$ & $a$ & $a$ & $a$ & $a$ & $\varangle$ & $\ll$ & $\varangle$ & $a_{1}$ & $a$ & $a$ & $a$ & $a$ & $a$ & $a$ & $a$ & $\ll$ \\
\hline$\dot{H}$ & $\stackrel{\oplus}{1}$ & $\stackrel{\mathscr{I}}{\mathscr{T}}$ & ॠี & $\stackrel{\mathscr{I}}{*}$ & है & $\stackrel{\mathscr{I}}{1}$ & Iี & $\stackrel{\mathscr{T}}{1}$ & $\stackrel{e}{\tilde{T}}$ & $\stackrel{\mathscr{I}}{\mathbb{1}}$ & $\stackrel{\mathscr{I}}{ }$ & $\stackrel{\mathscr{I}}{*}$ & $\stackrel{0}{\Psi}$ & 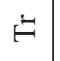 & 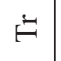 & $\stackrel{\mathscr{I}}{*}$ & है & $\stackrel{0}{ \pm}$ \\
\hline ల & లే & تَ & లี & $\stackrel{\ddot{I}}{1}$ & $\tilde{a}$ & $\stackrel{\mathscr{I}}{\mathbb{1}}$ & 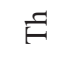 & $\tilde{F}$ & 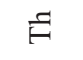 & $\stackrel{\ddot{I}}{\stackrel{0}{*}}$ & $\stackrel{\ddot{I}}{ }$ & $\stackrel{\ddot{I}}{\ddot{I}}$ & $\stackrel{0}{1}$ & $\tilde{D}$ & $\tilde{a}$ & U் & లే & 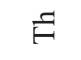 \\
\hline- & - & - & - & - & - & - & - & - & - & - & - & - & - & - & - & - & - & - \\
\hline$\theta$ & a & $\Sigma$ & D & D & D & $\theta$ & $\theta$ & $\theta$ & D & D & D & D & D & D & D & D & D & D \\
\hline Z & Z & Z & Z & Z & Z & Z & 띠 & Z & Z & Z & z & Z & Z & Z & Z & Z & Z & Z \\
\hline & & $\mathcal{U}$ & & & & & & & & & & & & & U & $\stackrel{U}{U}$ & & \\
\hline 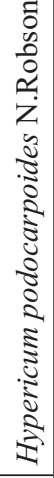 & 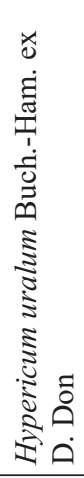 & 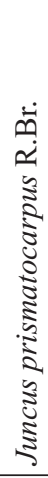 & 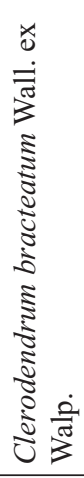 & 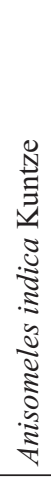 & 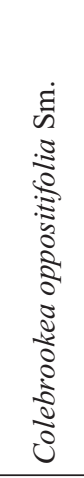 & 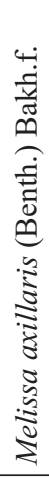 & 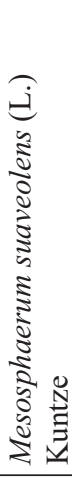 & 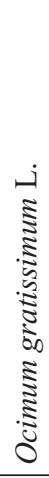 & 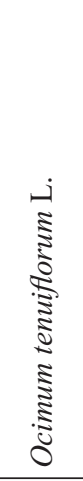 & 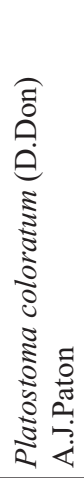 & 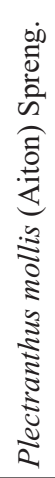 & 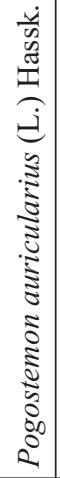 & 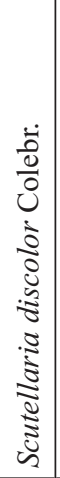 & 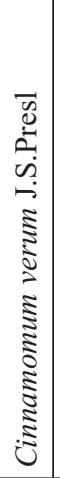 & 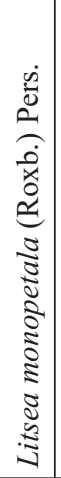 & 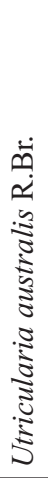 & 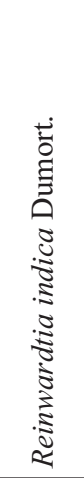 & 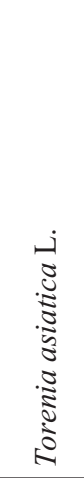 \\
\hline 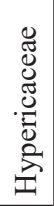 & 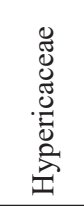 & 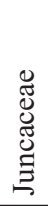 & 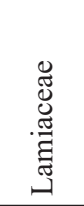 & 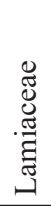 & 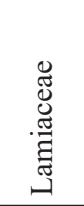 & 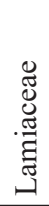 & 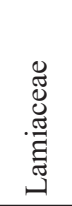 & 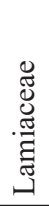 & 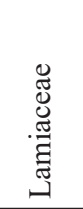 & 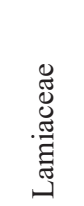 & 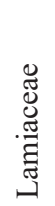 & 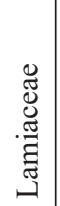 & 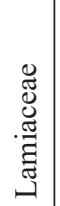 & 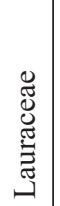 & 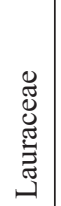 & 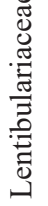 & 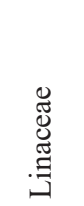 & 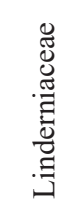 \\
\hline$\stackrel{\circ}{\circ}$ & $\hat{\circ}$ & $\stackrel{\infty}{\circ}$ & 。ิ & $\stackrel{\varrho}{=}$ & $\Xi$ & $\stackrel{\cong}{\Xi}$ & $\stackrel{\varrho}{=}$ & $\stackrel{\Xi}{\Xi}$ & $\cong$ & $\stackrel{0}{=}$ & $\cong$ & $\stackrel{\infty}{=}$ & $\triangleq$ & $\stackrel{ }{\beth}$ & $\vec{\beth}$ & ป & 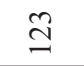 & $\stackrel{ \pm}{二}$ \\
\hline
\end{tabular}




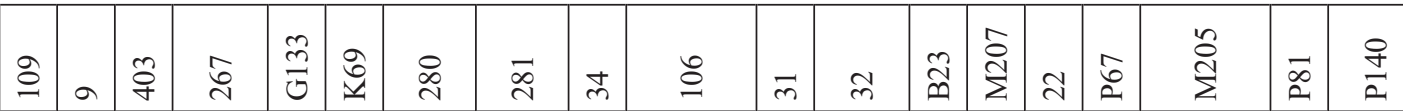

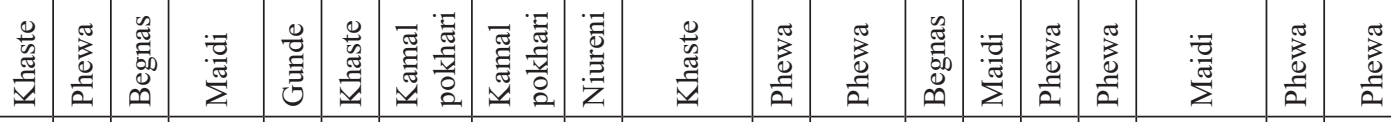

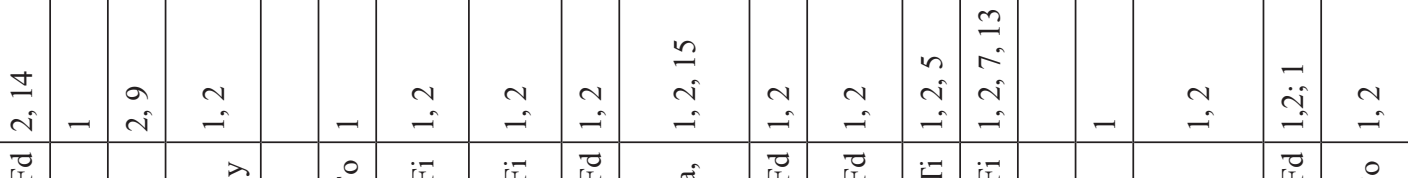

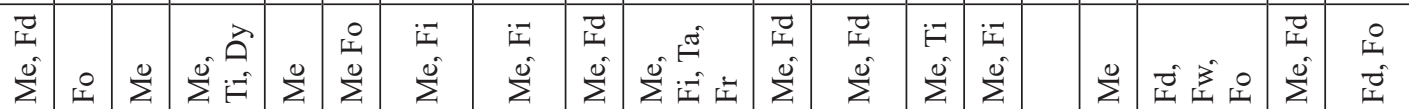
\begin{tabular}{llllllllllll|l|l|l|l|l|l}
\hline & $a$ & $a$ & $<$ & $a$ & $a$ & $a$ & $a$ & $a$ & $a$ & $a$ & $a$ & $a$ & $a$ & $a$ & $a$ & $a$ & $a$
\end{tabular} 至苗

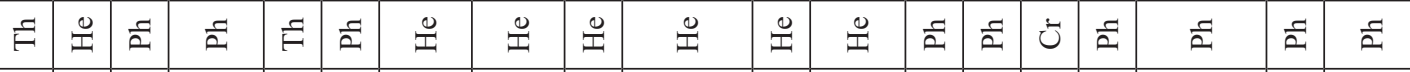

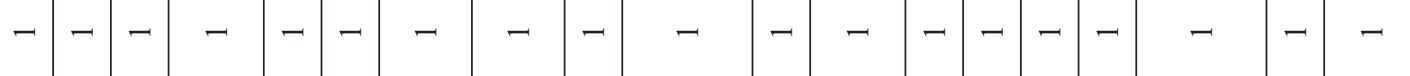

\begin{tabular}{|c|c|c|c|c|c|c|c|c|c|c|c|c|c|c|c|c|c|c|}
\hline D & D & D & D & D & D & ๑ & D & a & a & D & D & Q & $\theta$ & $\Sigma$ & $\theta$ & Q & D & $\theta$ \\
\hline Z & 디 & Z & Z & Z & 山匚 & Z & Z & Z & $\mathrm{Z}$ & Z & Z & Z & Z & Z & Z & Z & Z & Z \\
\hline
\end{tabular}

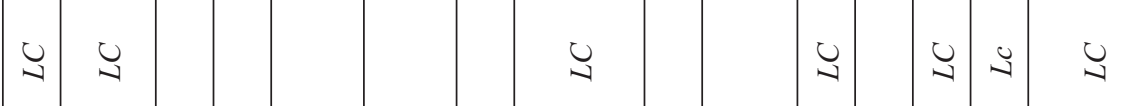

\begin{tabular}{|c|c|c|c|c|c|c|c|c|c|c|c|c|c|c|c|c|c|c|}
\hline 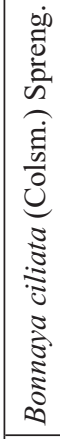 & 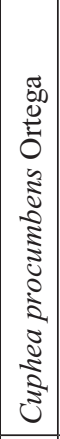 & 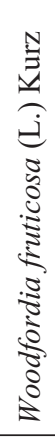 & 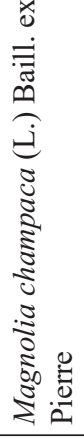 & 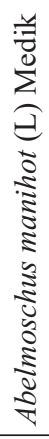 & 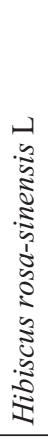 & 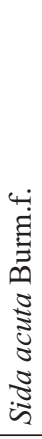 & 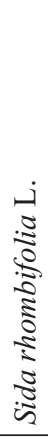 & 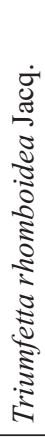 & $\begin{array}{l}\dot{i} \\
0 \\
0 \\
0 \\
0 \\
0 \\
0 \\
0 \\
0 \\
0\end{array}$ & 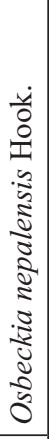 & 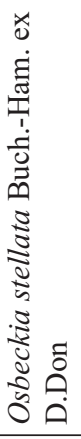 & 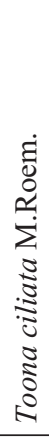 & 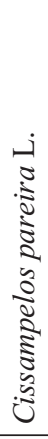 & 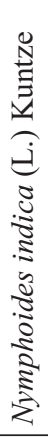 & 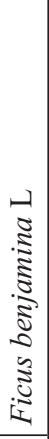 & 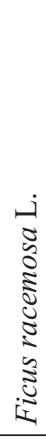 & 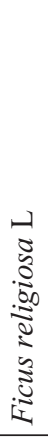 & 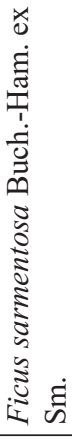 \\
\hline 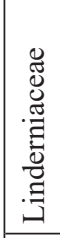 & 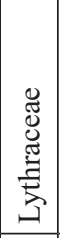 & 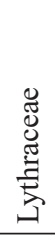 & 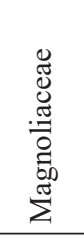 & 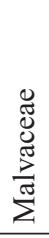 & 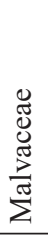 & 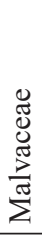 & 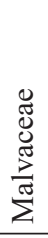 & 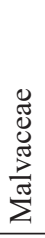 & 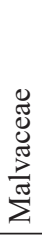 & 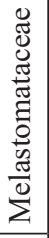 & 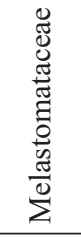 & 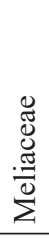 & 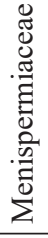 & 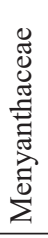 & 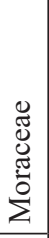 & 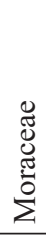 & 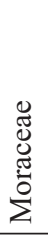 & 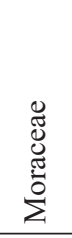 \\
\hline$\stackrel{\beth}{\beth}$ & $\stackrel{\bullet}{\sim}$ & $\widehat{\cong}$ & $\stackrel{\infty}{=}$ & さे & 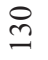 & $\bar{n}$ & $\cong$ & $\stackrel{m}{n}$ & $\stackrel{\oplus}{\sim}$ & $\stackrel{n}{n}$ & $\underset{ల}{\sim}$ & $\hat{n}$ & $\stackrel{\infty}{\sim}$ & ભે & \& & $\vec{\Xi}$ & I & $\stackrel{?}{\Xi}$ \\
\hline
\end{tabular}




\begin{tabular}{|c|c|c|c|c|c|c|c|c|c|c|c|c|c|c|c|c|}
\hline$\stackrel{2}{\not{1}}$ & $\stackrel{\widetilde{n}}{\oplus}$ & $\hat{\sigma}$ & $\underset{\sim}{+\infty}$ & $\begin{array}{l}\infty \\
\sim \\
N\end{array}$ & $\frac{\widetilde{I}}{2}$ & নิ & $\because$ & $\hat{a}$ & n & $\vec{n}$ & \begin{tabular}{l}
$\sum$ \\
\multirow{J}{\Xi}{}
\end{tabular} & రి & $\hat{n}$ & $\overline{\bar{N}} \sum_{\bar{\pi}}^{: \bar{\pi}}$ & $\approx$ & ñ \\
\hline$\stackrel{\widetilde{a}}{\stackrel{\Xi}{\mu}}$ & 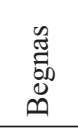 & 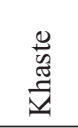 & $\stackrel{\widetilde{2}}{\stackrel{\Xi}{二}}$ & 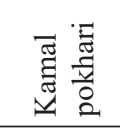 & $\frac{\pi}{2}$ & $\underset{\Xi}{\stackrel{\Xi}{二}}$ & 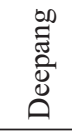 & $\sum_{\bar{\pi}}^{\bar{\pi}}$ & 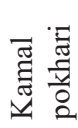 & 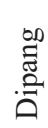 & $\sum_{\bar{\pi}}^{\frac{\pi}{\pi}}$ & : & $\underbrace{\bar{\pi}}_{\bar{\pi}}$ & : & $\frac{\pi}{2}$ & $\frac{\tilde{a}}{0}$ \\
\hline$\underset{\ddot{i}}{\ddot{i}}$ & $\begin{array}{l}m \\
i \\
i \\
-\end{array}$ & - & $\cong$ & $\begin{array}{l}0 \\
6 \\
\dot{0} \\
\therefore \\
-\end{array}$ & $\sim$ & $\stackrel{\sim}{\sim}$ & 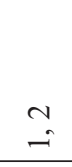 & $\stackrel{\sim}{\sim}$ & $N$ & & & & $\stackrel{\sim}{\cong}$ & & $\stackrel{\sim}{\sim}$ & $\begin{array}{l}6 \\
+5 \\
\therefore \simeq \\
-\infty \\
-\infty\end{array}$ \\
\hline 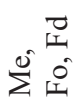 & $\begin{array}{l}\vec{I} \\
\dot{s}^{0}\end{array}$ & P & 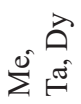 & $\vec{\nabla} \sum^{e} \vec{\alpha}$ & $\sum^{0}$ & $\sum^{0}$ & $\begin{array}{l}\ddot{\vec{\alpha}} \\
\sum^{0} \ddot{0}\end{array}$ & $\sum^{0}$ & $\sum^{\infty}$ & & & & $\sum^{m}$ & & $\sum^{0}$ & $\begin{array}{l}\sum_{i}^{0} \\
\dot{0}\end{array}$ \\
\hline$a$ & 0 & $a$ & $a$ & $a$ & $a$ & $a$ & $a$ & $a$ & $a$ & $a$ & $a$ & $a$ & $a$ & $a$ & 2 & $\varangle$ \\
\hline$\dot{H}$ & 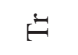 & $\dot{H}$ & 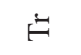 & $\stackrel{\oplus}{\Psi}$ & $\stackrel{\oplus}{I}$ & $\exists$ & $\stackrel{\mathscr{I}}{1}$ & $\stackrel{\oplus}{I}$ & $\stackrel{0}{I}$ & $\stackrel{\mathscr{I}}{ }$ & $\stackrel{0}{ \pm}$ & $\stackrel{\mathscr{I}}{1}$ & $\stackrel{\oplus}{1}$ & $\stackrel{\oplus}{1}$ & $\stackrel{0}{\mathbb{I}}$ & $\stackrel{\oplus}{I}$ \\
\hline$\frac{\tilde{Q}}{2}$ & $\tilde{a}$ & $\frac{\varepsilon}{2}$ & $\overline{2}$ & تَ & שٓ & $\bar{a}$ & Uี & $\stackrel{\oplus}{I}$ & $\tilde{U}$ & $\stackrel{\mathscr{I}}{I}$ & $\bar{a}$ & $\tilde{E}$ & $\bar{a}$ & $\dot{U}$ & $\stackrel{0}{I}$ & $\Xi$ \\
\hline- & - & - & - & - & - & - & - & - & - & - & - & - & - & - & - & - \\
\hline D & D & D & D & $\Sigma$ & $\Sigma$ & D & D & D & a & $\Sigma$ & $\Sigma$ & $\Sigma$ & $\Sigma$ & $\Sigma$ & D & D \\
\hline Z & Z & 띠 & Z & Z & Z & Z & Z & Z & 띠 & Z & Z & Z & z & Z & Z & 디 \\
\hline ن & & & $\underset{\exists}{U}$ & & $\underset{J}{U}$ & U & & $\underset{J}{U}$ & U & & & & & $\underset{J}{U}$ & $\underset{J}{U}$ & \\
\hline 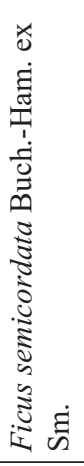 & 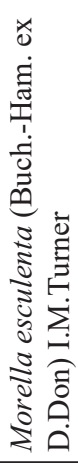 & 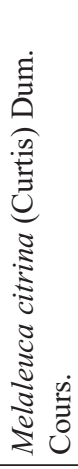 & 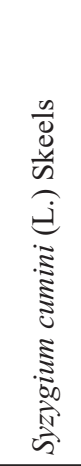 & 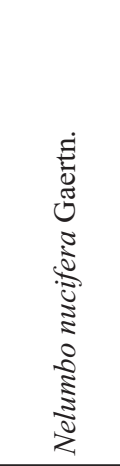 & 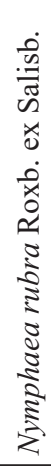 & 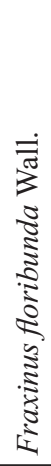 & 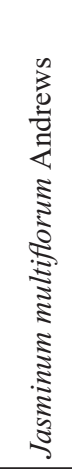 & 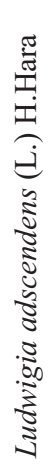 & 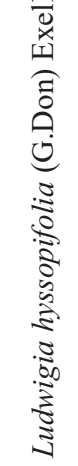 & 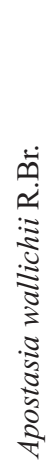 & 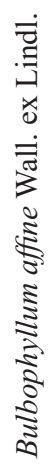 & 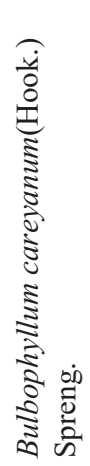 & 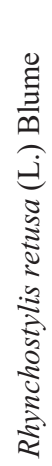 & 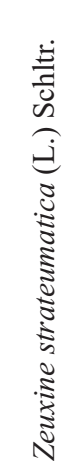 & 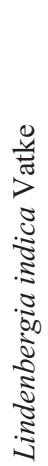 & 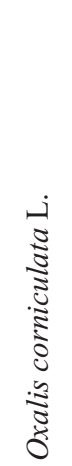 \\
\hline 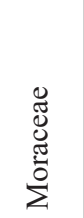 & 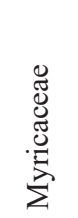 & 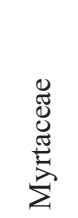 & 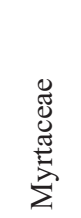 & 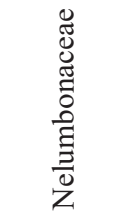 & 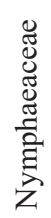 & $\begin{array}{l}\underset{\tilde{J}}{0} \\
\frac{\tilde{\Xi}}{0} \\
0\end{array}$ & 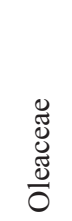 & 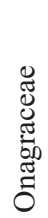 & 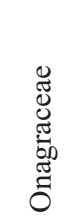 & 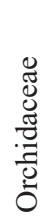 & 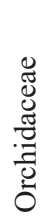 & 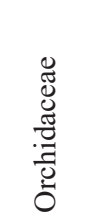 & 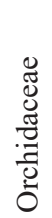 & 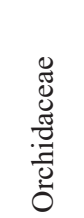 & 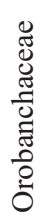 & 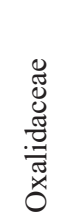 \\
\hline$\stackrel{\Xi}{ \pm}$ & 年 & 里 & 卞 & $\stackrel{\infty}{ \pm}$ & g & 은 & $\underline{n}$ & กี & $\hat{n}$ & $\stackrel{+}{n}$ & $n$ & $\stackrel{\circ}{2}$ & $\hat{n}$ & $\stackrel{\infty}{n}$ & 气ิ & $\stackrel{8}{0}$ \\
\hline
\end{tabular}




\begin{tabular}{|c|c|c|c|c|c|c|c|c|c|c|c|c|c|c|c|c|c|c|}
\hline$\stackrel{\vec{\sim}}{\vec{\Delta}}$ & $\begin{array}{l}\tilde{b} \\
\approx\end{array}$ & $\overrightarrow{\tilde{n}}$ & $\underset{n}{\stackrel{D}{~}}$ & $\stackrel{\sim}{m}$ & $\overline{6}$ & 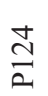 & $\stackrel{\infty}{+}$ & $\frac{\tilde{a}}{a}$ & ஜ & $\frac{\Delta}{m}$ & $\hat{\infty}$ & ๙n & $\stackrel{\sim}{\sim}$ & $\stackrel{\sim}{\sim}$ & $\stackrel{\overbrace{}}{\widetilde{U}}$ & $\overrightarrow{\widetilde{J}}$ & $\frac{n}{n}$ & $\underset{ల}{\stackrel{n}{n}}$ \\
\hline 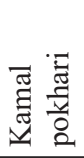 & 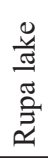 & 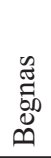 & $\begin{array}{l}\frac{\pi}{0} \\
\frac{3}{2} \\
\frac{\pi}{2}\end{array}$ & 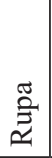 & 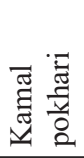 & $\frac{\pi}{2}$ & $\frac{\pi}{2}$ & $\frac{\pi}{0}$ & 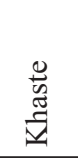 & $\stackrel{\widetilde{a}}{\vec{Z}}$ & $\frac{\pi}{0}$ & $\frac{\pi}{0}$ & 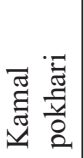 & 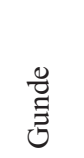 & 泀 & 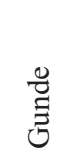 & 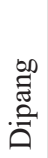 & $\frac{\pi}{0}$ \\
\hline & - & & $\begin{array}{l}\stackrel{1}{\sim} \\
\tilde{N}\end{array}$ & & & & & & & & $\stackrel{\sim}{-}$ & - & 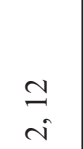 & & $\stackrel{\simeq}{\cong}$ & - & $\begin{array}{l}\simeq \\
\stackrel{\sim}{\sim}\end{array}$ & 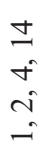 \\
\hline & $\vec{I}$ & $\sum^{\infty}$ & $\stackrel{n}{\Sigma}$ & & & & & & & & $\sum^{\infty}$ & 足 & $\sum^{\infty}$ & & 它 & $\overrightarrow{\mid \vec{y}}$ & $\sum^{0}$ & $\begin{array}{l}\vec{I} \\
\dot{\vec{\Sigma}}\end{array}$ \\
\hline$\ll$ & $a$ & a & $a$ & $a$ & $\varangle$ & $\ll$ & $a$ & $a$ & 2 & $a$ & $a$ & $a$ & $\varangle$ & $\varangle$ & $\varangle$ & $\ll$ & a & $a$ \\
\hline$\stackrel{0}{1}$ & 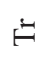 & 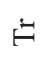 & 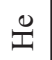 & ॠ & $\stackrel{0}{\dddot{I}}$ & $\stackrel{0}{I}$ & $\stackrel{\mathscr{I}}{\mathbb{I}}$ & $\stackrel{\oplus}{I}$ & $\stackrel{0}{I}$ & $\stackrel{0}{ \pm}$ & $\stackrel{\oplus}{I}$ & $\stackrel{0}{I}$ & $\stackrel{\mathscr{I}}{\stackrel{0}{*}}$ & 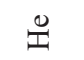 & $\stackrel{\oplus}{I}$ & $\stackrel{0}{I}$ & $\stackrel{\mathscr{I}}{\ddot{I}}$ & $\stackrel{0}{ \pm}$ \\
\hline$E$ & $\underline{a}$ & $\underline{a}$ & $\stackrel{0}{\dddot{I}}$ & 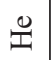 & 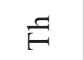 & 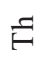 & تٓ & $\dot{U}$ & ت̇ & ت̇ & $\stackrel{\oplus}{I}$ & ت̇ & $E$ & 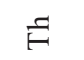 & $\stackrel{F}{F}$ & 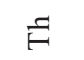 & $\dot{U}$ & $\dot{U}$ \\
\hline- & - & - & - & - & - & - & - & - & - & - & - & - & - & - & - & - & - & $\nabla$ \\
\hline D & D & D & A & D & A & D & $\Sigma$ & $\Sigma$ & $\Sigma$ & $\Sigma$ & $\Sigma$ & $\Sigma$ & $\Sigma$ & $\Sigma$ & $\Sigma$ & $\Sigma$ & $\Sigma$ & $\Sigma$ \\
\hline 디 & Z & Z & Z & Z & Z & Z & Z & Z & 디 & Z & Z & Z & Z & Z & Z & Z & Z & [工] \\
\hline & & $\underset{\sim}{U}$ & & & $\underset{\sim}{\cup}$ & $\underset{\sim}{U}$ & & & $\underset{\sim}{\cup}$ & & & & $\underset{\sim}{\cup}$ & $\underset{\sim}{U}$ & & $\underset{\exists}{\cup}$ & & $\underset{\checkmark}{U}$ \\
\hline 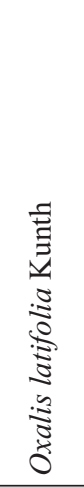 & 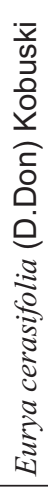 & 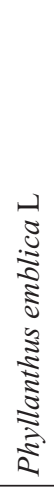 & 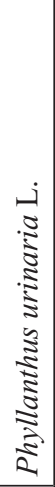 & 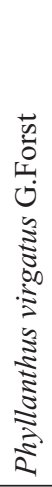 & 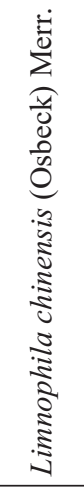 & 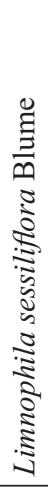 & 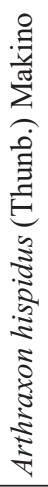 & 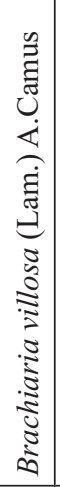 & 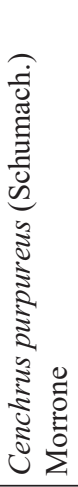 & 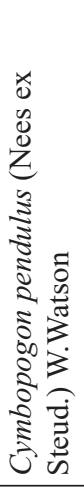 & 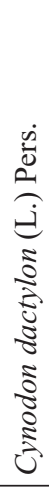 & 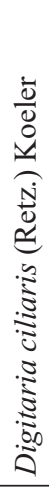 & 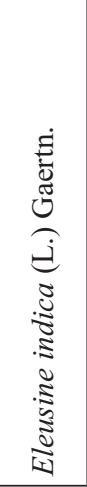 & 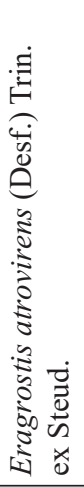 & 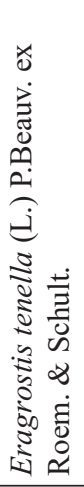 & 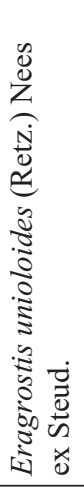 & 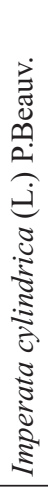 & 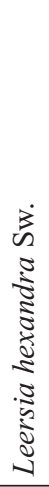 \\
\hline 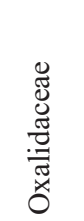 & 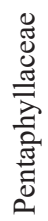 & 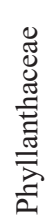 & 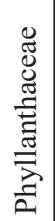 & 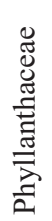 & 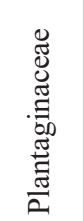 & 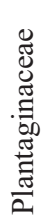 & 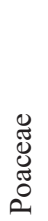 & 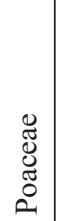 & 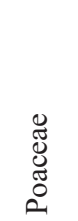 & 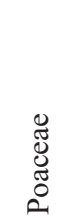 & $\begin{array}{l}\mathscr{\Xi} \\
\stackrel{\Xi}{0} \\
0 \\
0\end{array}$ & 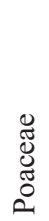 & 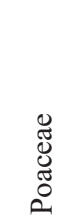 & 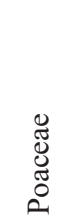 & 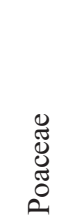 & 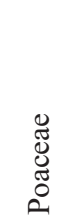 & 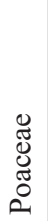 & 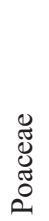 \\
\hline $\bar{\sigma}$ & $\widetilde{\sigma}$ & $\underline{6}$ & $\underset{-}{\mathbb{0}}$ & $\sqrt{6}$ & $\stackrel{0}{0}$ & $\underline{\sigma}$ & $\stackrel{\infty}{\infty}$ & bิ & $\stackrel{P}{I}$ & $\Xi$ & $\cong$ & $\stackrel{\cong}{\beth}$ & $\stackrel{ \pm}{ \pm}$ & $\stackrel{n}{\simeq}$ & $\stackrel{\circ}{\beth}$ & 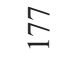 & $\stackrel{\infty}{=}$ & $\stackrel{2}{ }$ \\
\hline
\end{tabular}


28 Pathak et al.: Floristic Diversity in the Lake Cluster of Pokhara Valley, Central Nepal

\begin{tabular}{|c|c|c|c|c|c|c|c|c|c|c|c|c|c|c|c|c|c|}
\hline$\infty$ & $\stackrel{\circ}{\sim}$ & in & $\stackrel{\wp}{f}$ & $\frac{2}{0}$ & $\hat{a}$ & $\stackrel{M}{\Xi}$ & $\ddot{\sim}$ & તิ & $\stackrel{゚}{\stackrel{0}{n}}$ & $\stackrel{\text { I }}{m}$ & $\vec{\sim}$ & $\stackrel{0}{\sim}$ & in & $\vec{\sim}$ & $\mathbb{N}$ & $\underset{\Xi}{\Xi}$ & $\vec{m}$ \\
\hline$\frac{\pi}{0}$ & $\begin{array}{c}\frac{\pi}{2} \\
\frac{2}{2} \\
\frac{1}{2}\end{array}$ & $\begin{array}{c}\frac{\pi}{3} \\
\frac{\pi}{2} \\
\frac{\pi}{2}\end{array}$ & $\frac{\pi}{2}$ & $\frac{\pi}{0}$ & 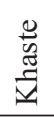 & 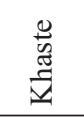 & $\frac{\pi}{2}$ & 总 & 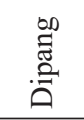 & 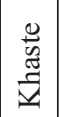 & $\frac{\pi}{0}$ & $\stackrel{\tilde{a}}{\vec{a}}$ & $\frac{\pi}{0}$ & $\frac{\pi}{\frac{\pi}{2}}$ & 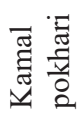 & $\stackrel{:=\bar{\pi}}{\sum_{\Sigma}^{\pi}}$ & 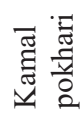 \\
\hline- & & - & $a$ & $\begin{array}{l}\hat{\imath} \\
\hat{\imath}\end{array}$ & - & $\begin{array}{l}\cong \\
\cong \\
\cong\end{array}$ & & & $\vec{\sim}$ & $\stackrel{\sim}{\sim}$ & $\begin{array}{l}0 \\
\hat{0} \\
\tilde{\sigma}\end{array}$ & $\stackrel{\sim}{\sim}$ & $\begin{array}{l}\sigma \\
\forall i n \\
i \pm \\
\therefore \pm\end{array}$ & & $\stackrel{\sim}{\sim}$ & & $\stackrel{\sim}{\sim}$ \\
\hline i & & $\dot{\overrightarrow{\mid}}$ & 它 & $\sum_{\overrightarrow{1}+}^{0}$ & $\dot{15}$ & 它 & & & 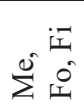 & $\sum^{0}$ & 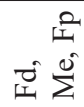 & $\begin{array}{l}0 \\
\dot{\Sigma} \\
\dot{\Sigma}\end{array}$ & $\begin{array}{l}\text { से } \\
\stackrel{2}{\Sigma^{\circ}}\end{array}$ & & 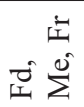 & & $\sum_{i=1}^{0}$ \\
\hline$\varangle$ & $a$ & $\varangle$ & $a$ & $\varangle$ & $\varangle$ & $a$ & $a$ & 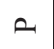 & $a$ & $\varangle$ & $a$ & $a$ & $\varangle$ & $\varangle$ & $\varangle$ & $\varangle$ & $\varangle$ \\
\hline$\stackrel{\mathscr{I}}{\stackrel{0}{1}}$ & $\stackrel{0}{I}$ & $\stackrel{0}{ \pm}$ & $\stackrel{0}{\mathbb{I}}$ & $\stackrel{0}{I}$ & $\stackrel{0}{\mathbb{I}}$ & $\stackrel{0}{I}$ & $\stackrel{\mathscr{I}}{ }$ & 寽 & ॠే & $\stackrel{\mathscr{I}}{\mathbb{1}}$ & $\stackrel{\oplus}{1}$ & $\stackrel{\mathscr{I}}{ }$ & $\stackrel{\mathscr{I}}{ }$ & $\stackrel{\oplus}{1}$ & $\stackrel{0}{I}$ & $\stackrel{\mathscr{I}}{ }$ & $\stackrel{\mathscr{I}}{ }$ \\
\hline$E$ & تَّ & $\Xi$ & تَ & $\Xi$ & $E$ & تَ & $\dot{U}$ & $\dot{U}$ & $\stackrel{0}{I}$ & $E$ & $\stackrel{\ddot{I}}{ }$ & $\dot{U}$ & 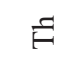 & $E$ & $\Xi$ & $E$ & $\tilde{F}$ \\
\hline- & - & - & - & - & - & - & - & - & - & - & - & - & - & - & - & - & $\nabla$ \\
\hline$\Sigma$ & $\Sigma$ & $\Sigma$ & $\Sigma$ & $\Sigma$ & $\Sigma$ & $\Sigma$ & $\Sigma$ & $\Sigma$ & $\Sigma$ & D & D & D & a & D & D & D & D \\
\hline Z & Z & Z & 디 & Z & Z & Z & Z & Z & Z & Z & Z & Z & Z & Z & Z & Z & Z \\
\hline & $\underset{\exists}{U}$ & & & $\underset{J}{U}$ & U & & & & & & $\underset{V}{U}$ & & $\underset{J}{U}$ & $\mathcal{U}$ & & & \\
\hline 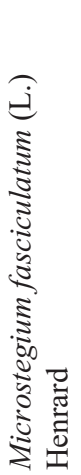 & 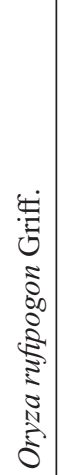 & 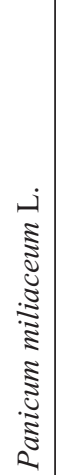 & 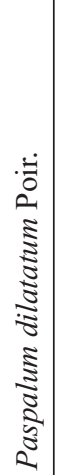 & 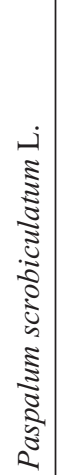 & 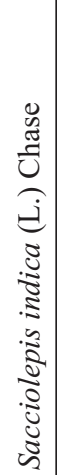 & 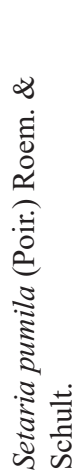 & 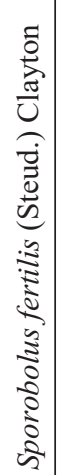 & 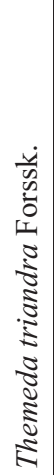 & 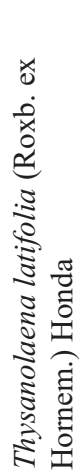 & 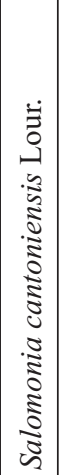 & 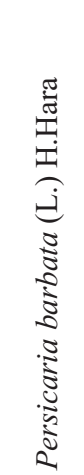 & 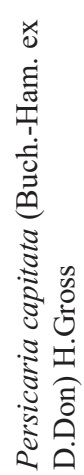 & 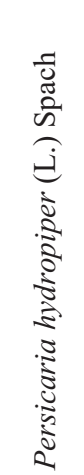 & 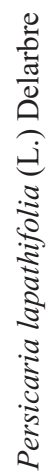 & 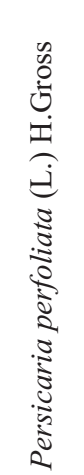 & 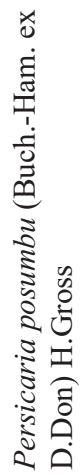 & 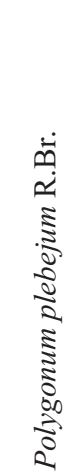 \\
\hline 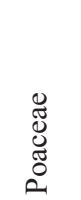 & $\begin{array}{l}\mathscr{J} \\
\stackrel{J}{0} \\
\stackrel{0}{0}\end{array}$ & 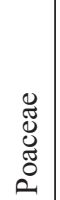 & 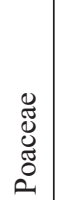 & $\begin{array}{l}\mathscr{\Xi} \\
\mathbb{\Xi} \\
\tilde{\Xi} \\
0\end{array}$ & 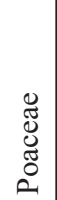 & $\begin{array}{l}\mathscr{J} \\
\stackrel{\Xi}{0} \\
\stackrel{\Xi}{0} \\
\stackrel{0}{0}\end{array}$ & 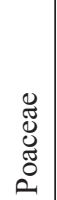 & 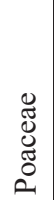 & $\begin{array}{l}\mathscr{J} \\
\mathbb{\Xi} \\
0 \\
0\end{array}$ & 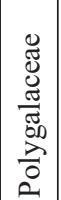 & 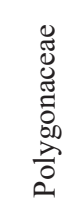 & 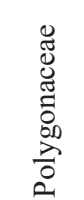 & 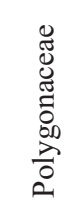 & 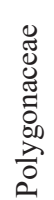 & 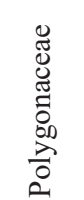 & 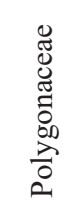 & 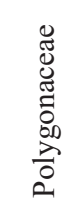 \\
\hline$\stackrel{\infty}{\infty}$ & 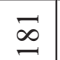 & $\underset{\infty}{\infty}$ & $\stackrel{\infty}{\infty}$ & $\underset{-}{+}$ & $\infty$ & $\infty$ & $\hat{\infty}$ & $\begin{array}{l}\infty \\
\infty\end{array}$ & $\infty$ & 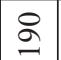 & $\bar{a}$ & $\Omega$ & 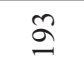 & す & $\stackrel{\curvearrowleft}{\varrho}$ & $\stackrel{\circ}{\circ}$ & $\hat{a}$ \\
\hline
\end{tabular}


Journal of Natural History Museum Volume 31, 2019-20 29

\begin{tabular}{|c|c|c|c|c|c|c|c|c|c|c|c|c|c|c|c|c|c|c|}
\hline$\curvearrowleft$ & $\bar{m}$ & ป & $n$ & $\frac{\widetilde{\sigma}}{\widetilde{\alpha}}$ & $\sum^{\infty}$ & $\stackrel{\infty}{\sim}$ & $\underset{ల}{0}$ & $\stackrel{\varrho}{\equiv}$ & $\stackrel{\overbrace{}}{\stackrel{\overbrace{}}{\Sigma}}$ & $\underset{d}{d}$ & $\stackrel{\sim}{\infty}$ & હิ & $\stackrel{-}{=}$ & $\hat{\sigma}$ & $\sum_{\Sigma}^{\infty}$ & $\begin{array}{l}\hat{\sigma} \\
\widetilde{a}\end{array}$ & 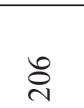 & 우 \\
\hline 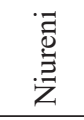 & $\frac{\pi}{2}$ & 泀 & 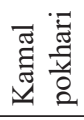 & $\stackrel{\widetilde{2}}{\stackrel{\Xi}{\Xi}}$ & : & 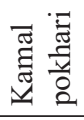 & $\frac{\tilde{3}}{0}$ & 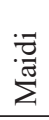 & $\sum_{\bar{\pi}}^{\frac{\pi}{\pi}}$ & $\underset{\check{Z}}{\stackrel{\Xi}{二}}$ & $\begin{array}{l}\tilde{Z} \\
\tilde{0} \\
\tilde{D} \\
\tilde{D}\end{array}$ & 泀 & $\frac{\pi}{3}$ & $\frac{\pi}{2}$ & $i_{\bar{\Xi}}^{\bar{\Xi}}$ & 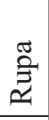 & $\begin{array}{l}\frac{\mathbb{U}}{\tilde{Z}} \\
\stackrel{\Xi}{\Xi}\end{array}$ & $\frac{\pi}{0}$ \\
\hline$\stackrel{\sim}{-}$ & $\stackrel{\sigma}{\sigma}$ & $\begin{array}{l} \pm \\
\stackrel{\sim}{-}\end{array}$ & & $\stackrel{\sim}{-}$ & - & $\begin{array}{l}\hat{b} \\
\hat{0} \\
\dot{0} \\
\hat{i} \\
\hat{-}\end{array}$ & $\stackrel{\sim}{\sim}$ & & $\stackrel{\sim}{\cong}$ & 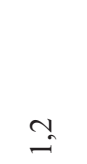 & $\stackrel{\sim}{\cong}$ & & $\cong$ & & - & & & $ㅇ$ \\
\hline$\vec{I}$ & శే & $\sum_{i=1}^{0}$ & & $\stackrel{n}{\Sigma}$ & 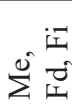 & $\sum_{i=1}^{0}$ & 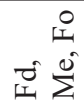 & & $\sum_{i=1}^{0}$ & $\dot{\vec{\Sigma}}$ & $\begin{array}{l}0 \\
\stackrel{1}{0}^{0}\end{array}$ & & $\sum^{0}$ & & $\sum^{0}$ & & & $\sum^{0}$ \\
\hline$a$ & $a$ & $a$ & $a$ & $a$ & $a$ & $\varangle$ & $a$ & $a$ & $a$ & $a$ & $a$ & $a$ & $\varangle$ & $a$ & $a$ & $\varangle$ & $a$ & $a$ \\
\hline$\stackrel{\mathscr{I}}{\mathbb{1}}$ & $\stackrel{\mathscr{I}}{\stackrel{0}{*}}$ & $\stackrel{\oplus}{I}$ & $\stackrel{0}{I}$ & ॠี & ॠ & $E$ & ॠี & ๘ & $\stackrel{\mathscr{I}}{\stackrel{0}{*}}$ & ๘ี & $\stackrel{\mathscr{I}}{1}$ & $\stackrel{\mathscr{I}}{\stackrel{0}{*}}$ & $\stackrel{0}{I}$ & $\stackrel{0}{I}$ & ॠ & $\stackrel{0}{\stackrel{I}{*}}$ & $\stackrel{0}{I}$ & $\stackrel{\mathscr{I}}{I}$ \\
\hline$\stackrel{\oplus}{I}$ & تَ & $\dot{U}$ & تَ & $\tilde{a}$ & లే & $\Xi$ & $\tilde{U}$ & $\tilde{U}$ & $\stackrel{0}{I}$ & $\tilde{U}$ & $\stackrel{0}{I}$ & $\stackrel{0}{I}$ & 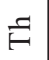 & $\stackrel{0}{I}$ & $\tilde{U}$ & $\stackrel{F}{F}$ & $\stackrel{\oplus}{I}$ & $\stackrel{\mathscr{I}}{ }$ \\
\hline- & - & - & - & - & - & & - & - & - & - & - & - & - & - & - & - & - & - \\
\hline D & $\Sigma$ & $\Sigma$ & $\Sigma$ & a & a & D & D & D & a & a & D & a & a & D & a & A & $\theta$ & a \\
\hline Z & 山 & Z & Z & Z & Z & Z & Z & Z & Z & Z & Z & Z & Z & Z & Z & Z & Z & 山 \\
\hline & & & & & & $\underset{\checkmark}{U}$ & $\underset{\checkmark}{U}$ & & & & & & & & & $\underset{J}{U}$ & & \\
\hline 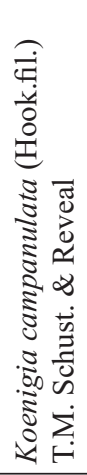 & 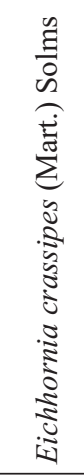 & 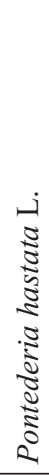 & 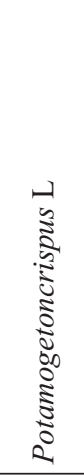 & 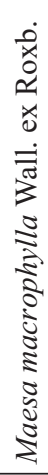 & 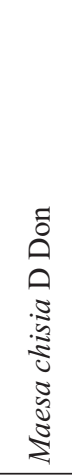 & 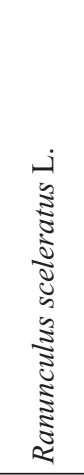 & 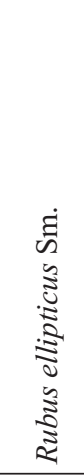 & 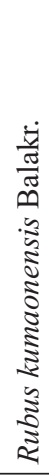 & 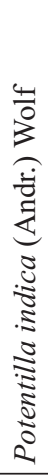 & 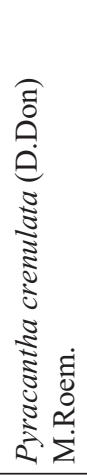 & 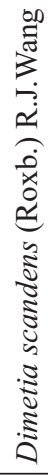 & 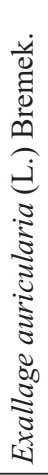 & 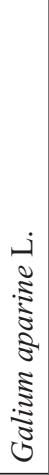 & 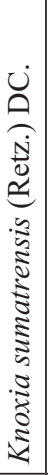 & 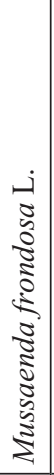 & 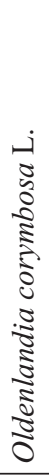 & 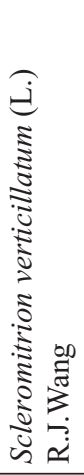 & 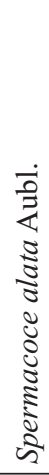 \\
\hline 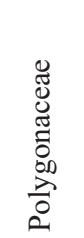 & 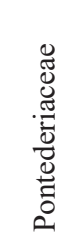 & 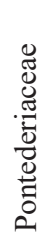 & 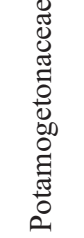 & 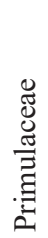 & 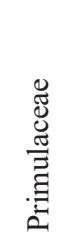 & 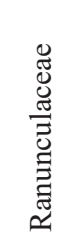 & 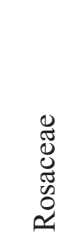 & $\begin{array}{l}\mathscr{J} \\
\mathbb{J} \\
\tilde{J} \\
\tilde{0} \\
\mathscr{0}\end{array}$ & 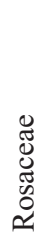 & 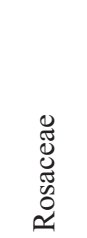 & 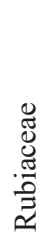 & 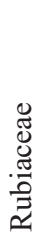 & 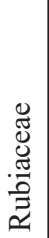 & 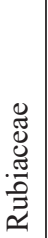 & 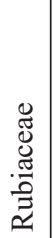 & 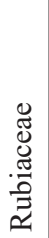 & 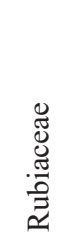 & 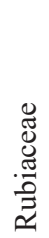 \\
\hline$\stackrel{\infty}{\varrho}$ & Ә & ఠి & 。্ & ฮิ & กิ & ¿্ & ڤ̊ & 로 & હ) & $\stackrel{\infty}{\stackrel{\nu}{~}}$ & 우 & $\stackrel{ }{\sim}$ & $\bar{\sim}$ & $\frac{\stackrel{N}{N}}{2}$ & $\frac{m}{\sim}$ & $\underset{\sim}{\Delta}$ & $\stackrel{n}{\sim}$ & $\stackrel{0}{\sim}$ \\
\hline
\end{tabular}


30 Pathak et al.: Floristic Diversity in the Lake Cluster of Pokhara Valley, Central Nepal




Appendix II

\begin{tabular}{|c|l|}
\hline Code & References \\
\hline 1 & MANANDHAR, N P (2002) Plants and people of Nepal. Timber press. \\
\hline 2 & $\begin{array}{l}\text { BARAL, S R; KURMI, P P(2006)A compendium of medicinal plants in } \\
\text { Nepal. Kathmandu, Nepal: Rachana Sharma. }\end{array}$ \\
\hline 3 & $\begin{array}{l}\text { BISHOKARMA, B K; KINSEY, C K, DANGOL, D R; CHAUDHARY, P } \\
\text { (2005) Folk use of plant resource at Madi valley of Chitwan District, Nepal. } \\
\text { Banko Janakari 15(2):28-33. }\end{array}$ \\
\hline 4 & $\begin{array}{l}\text { JOSHI, A R; JOSHI, K (2009) Indigenous Uses of Wetland Plant Diversity } \\
\text { of two Valleys (Kathmandu and Pokhara) in Nepal. Ethnobotany 21: 11-17 }\end{array}$ \\
\hline 5 & $\begin{array}{l}\text { ACHARYA, KP(2009) Utilization and status of plant resources in northern } \\
\text { Part of Pokhara Valley, Central Nepal. Ethnobotanical leaflets 2009(1): } 13 .\end{array}$ \\
\hline 6 & $\begin{array}{l}\text { LAMSAL, P; PANT, K P; KUMAR, L; ATREYA, K (2014)Diversity, uses, } \\
\text { and threats in the Ghodaghodi Lake Complex, a Ramsar site in western } \\
\text { lowland Nepal. ISRN Biodiversity, 2014. }\end{array}$ \\
\hline 7 & $\begin{array}{l}\text { ADHIKARI, M; THAPA, R; KUNWAR, R M; DEVKOTA, H P; POUDEL, } \\
\text { P (2019) Ethnomedicinal Uses of Plant Resources in the Machhapuchchhre } \\
\text { Rural Municipality of Kaski District, Nepal. Medicines 6(2): 69. }\end{array}$ \\
\hline 10 & $\begin{array}{l}\text { KUNWAR, R M; BURLAKOTI, C; CHOWDHARY, C L; BUSSMANN, } \\
\text { properties, chemical constituents, and biological activities. Pharmacognosy } \\
\text { pharmacological validity. Med Aromat Plant Sci Biotechnol 4(1): 28-42. }\end{array}$ \\
\hline Slants in Ghodaghodi lake area.Nepal. J Nat Hist Mus 21(1-4): 243-66. \\
\hline
\end{tabular}




\begin{tabular}{|c|l|}
\hline 11 & $\begin{array}{l}\text { BUDHA-MAGAR, S; BHANDARI, P; GHIMIRE, S K (2020) Ethno- } \\
\text { medicinal survey of plants used by Magar (Kham) community, Rolpa } \\
\text { district, Western Nepal. Ethnobotany Research and Applications, 19:1-29. }\end{array}$ \\
\hline 12 & $\begin{array}{l}\text { BHATT, M. D; KUNWAR, R. M. (2020). Distribution pattern and } \\
\text { ethnomedicinal uses of plants in Kanchanpur district, Far-Western Nepal. } \\
\text { Ethnobotany Research and Applications 20:1-21. }\end{array}$ \\
\hline 13 & $\begin{array}{l}\text { KUNWAR, R M; ACHARYA, R P; CHOWDHARY, C L; BUSSMANN, } \\
\text { R W (2015) Medicinal plant dynamics in indigenous medicines in farwest } \\
\text { Nepal. Journal of Ethnopharmacology 163: 210-219. }\end{array}$ \\
\hline 14 & $\begin{array}{l}\text { NIROULA, B; SINGH, K L B (2011) Aquatic plant resources of Betana } \\
\text { Wetland, Morang, Nepal. Our Nature, 9(1): 146-155. }\end{array}$ \\
\hline 15 & $\begin{array}{l}\text { SHARMA, K; SAUD, D S; BHATTARAI K R; DHAKAL, S; KHADKA, } \\
\text { M K (2020) Wetland Plants and their Ethnobotanical Uses in Raja-Rani Tal, } \\
\text { Letang, Morang, Nepal. J. Pl. Res. 18(1):135-142 }\end{array}$ \\
\hline
\end{tabular}

